\title{
Article \\ Application of the Shannon Entropy in the construction of a Paraconsistent Model of the Atom
}

\author{
João Inácio da Silva Filho * \\ Laboratory of Applied Paraconsistent Logic, Santa Cecilia University \\ Oswaldo Cruz Street, 288, Santos City, SP, Brazil, 11045-000 \\ * Correspondence: inacio@unisanta.br
}

\begin{abstract}
In this work, we present a model of the atom that is based on nonclassical logic called paraconsistent logic (PL), which has the main property of accepting the contradiction in logical interpretations without the conclusions being annulled. The model proposed in this work is constructed with the extension of PL called paraconsistent annotated logic with annotation of two values (PAL2v) that is associated with an interlaced bilattice of four vertices. We used the logarithmic function of the Shannon entropy $H_{(s)}$ with the inclusion of the normalized Planck constant $\hbar$ to construct the paraconsistent equations. Through the analyses of the interlaced bilattice, comparative values are obtained for some of the phenomena and effects of quantum mechanics, such as superposition of states, quantum entanglement, wave functions, and equations that determine the energy levels of the layers of the atom. At the end of this article, we use the hydrogen atom as the basis of the representation of the PAL2v model, where the values of the energy levels in six orbital layers are obtained. As an example, we present a possible method of applying the PAL2v model to the use of Raman spectroscopy signals in quality detection of lubricating mineral oil.
\end{abstract}

Keywords: quantum information; Shannon entropy; quantum physics; paraconsistent logic; mathematics and computing

\section{Introduction}

The model of the atom was presented by Niels Bohr in 1913, where he proposed that electrons are particles with two kinds of motion in atoms. In the Bohr model, the electrons either move continuously around the nucleus in certain stationary orbits or discontinuously jump between these orbits [1]. Subsequently, with the advances in quantum theory, new concepts, such as the ideas of superposition of states and quantum entanglement, have been proposed. Currently, the physical state of an electron is described by a wave function $[1,2]$.

In the foundations of quantum mechanics, the wave function is a description of the random discontinuous motion of particles. Moreover, the data on the physical properties of particles are uncertain and all of the analyses are probabilistic. The probability density of the particle appearing in each position is proportional to the square of the modulus of its wave function at every instant. The square of the modulus of the wave function represents not only the probability of a particle being found at a certain location but also the probability of the particle being there [3][4][5]. In 1925, Heisenberg published results introducing the quantum concepts for particles in matrix analysis. In the matrix formulation, the instantaneous state of a quantum system encodes the probabilities of its measurable properties or "observables" that include energy, position, momentum, and angular momentum. Observables can be either continuous (e.g., the position of a particle) or discrete (e.g., the energy of an electron bound to a hydrogen atom). 
In 1926, Schrödinger proposed a partial differential equation for the wave functions of particles, such as electrons. The state of a system at a given time is described by a complex wave function, which is also referred to as the state vector in a complex vector space, and this abstract mathematical object enables the calculation of the probabilities of outcomes of concrete experiments. Another important consideration is that, in quantum mechanics, one can never make simultaneous predictions of conjugate variables, such as position and momentum, to arbitrary precision.

In 1927, Heisenberg proposed the uncertainty principle, which states the formal inequality relating the uncertainty of position $\Delta_{x}$ and the uncertainty of momentum $\Delta_{p}$, as follows $[3,4]$ :

$$
\Delta_{x} \Delta_{p} \geq \frac{\hbar}{2}
$$

The electrons may be considered (to a certain probability) to be located somewhere within a given region of space. However, their exact positions are unknown. In this condition, contours of constant probability density, which are often referred to as "clouds," may be drawn around the nucleus of an atom to conceptualize where the electron might be located with the most probability [2][5-8]. The probability density is obtained using the square of the amplitude of the wave function, which usually involves a complex quantity. Thus, its value is derived by multiplication with the conjugate complex, as follows [6,7]:

$$
D p=\psi_{(x, t)}^{*} \psi_{(x, t)} \cdot
$$

If the wave function is the representative of the sum of probabilities that describe a particle, then it needs to be normalized, as follows [8]: $\int_{-\infty}^{+\infty} \psi^{*}(x, t) \psi_{(x, t)} d x=1$.

With respect to the logic applied to quantum mechanics among various studies of quantum probabilistic logic formalism, one of the most important was developed by von Neumann in 1932 [11,12]. In his work, von Neumann assumed that each physical system is associated with a Hilbert space $H$ (separable), with its unit vectors corresponding to possible physical states of the system. Each real "observable" random quantity is represented by a self-regulated operator $A$ in $H$ whose spectrum is the set of possible values of $A$ [13-16]. According to these previous works, mathematics in quantum mechanics can be considered a nonclassical probability calculation, which is supported by a nonclassical propositional logic $[17,18]$.

Nonclassical logics are created with the purpose of opposing the binary principles of classical logic, thus providing better conditions for the construction of physical-mathematical models with more approximate results. Currently, there are several types of nonclassical logics, and in general, we can consider that only those logics that are indestructible in the presence of the contradiction receive the denomination of paraconsistent logic (PL). Therefore, PL is a nonclassical logic that has, as its fundamental characteristic, the opposition to the principle of noncontradiction [19-21].

The fundamental theory of PL has been developed in the area of philosophical logic [23], and a formal framework for inconsistent theories was proposed by da Costa [20]. Further details of the logical formalization of PL, the mathematical implications, and their theorems can be found in [10], [22], and [23].

Blair and Subrahmanian [24] presented applications of PL to logical programming and extended the formalization of three-valued semantics. With this initial work, a theory was developed for possibly inconsistent logic programs using a lattice also known as Belnap's four-valued logic [25]. Such a lattice can be used for the associated lattice of paraconsistent annotated logic with annotation of two values (PAL2v). In Belnap's four-valued logic [25], the set of truth values of four-valued logic is defined as $\tau=\{t, f, \mathrm{~T}, \perp\}$, in which $t, f, \mathrm{~T}, \perp$ are propositions in the language of a program that denote true, false, contradictory, and paracomplete, 
respectively. The set of truth values $\tau$ comprises a complete lattice under the ordering $\leq$, such that $\perp \leq x \geq \mathrm{T}$ for $x € \tau=\{t, f\}[24,25]$.

The PL, particularly in its extended form called paraconsistent annotated logic (PAL) that has an associated lattice, has been investigated and applied to several fields of science [26]. The first concepts of PAL2v, which can be applied to artificial intelligence, were presented in [27]. As presented in [27], [28], [29], and [30], in the application of PAL2 $\mathrm{v}$, the associated lattice is considered an abstract universe $\tau$, where a negation operator allows logical interpretations to result in paraconsistent equations. In data analysis systems, the PAL can derive an annotation composed of two degrees of evidence from different sources of information. In the annotation, the first degree of evidence is favorable for the proposition $P$ and represented by the symbol $\mu$, and the second degree of evidence is unfavorable for the proposition $P$ and represented by the symbol $\lambda$. These degrees of evidence are normalized, classified as a set of real numbers, and contained in the closed interval $[0,1]$. The annotation assigns a logical state to the proposition. Thus, the information in PAL2v is a paraconsistent logical signal represented by the proposition $P$ with the subscript of the annotation $(\mu, \lambda): P_{(\mu, \lambda)}$, where the annotation pair is composed of the degrees of favorable evidence $(\mu)$ and unfavorable evidence $(\lambda)$.

The paraconsistent symbol $(\mu, \lambda)$ assigns a logical state to proposition $P$, as follows [27][30]:

1. If the annotation is $(0,1)$, then the degree of favorable evidence is minimum and the degree of unfavorable evidence is maximum, which provides a logical "false" connotation to proposition $P$. This paraconsistent signal defines the logical state "false" $f$.

2. If the annotation is $(1,0)$, then the degree of favorable evidence is maximum and the degree of unfavorable evidence is minimum, which provides a logical "true" connotation to proposition $P$. This paraconsistent signal defines the logical state "true" $t$.

3. If the annotation is $(1,1)$, then the degree of favorable evidence is maximum and the degree of unfavorable evidence is maximum, which provides a logical true and false connotation to proposition $P$. This paraconsistent signal defines the logical state "inconsistent" $T$.

4. If the annotation is $(0,0)$, then the degree of favorable evidence is minimum and the degree of unfavorable evidence is minimum, which provides a logical false and true connotation to proposition $P$. This paraconsistent signal defines the logical state "paracomplete" $\perp$.

Fig. 1(a) shows the lattice FOUR associated with PAL and the representations of the extreme logical states in their vertices through the annotation $(\mu, \lambda)$ of PAL2v $[27,28]$.

As discussed in [31], [32], and [33], this representation of PAL2v has been recently investigated using an interlaced bilattice also known as bilattice of Belnap [25][34]. A bilattice is a structure $B=\left\langle B, \leq_{t}, \leq_{k}\right\rangle$, where $B$ is a non-empty set and $\left\langle B, \leq_{k}\right\rangle$ and $\left\langle B, \leq_{t}\right\rangle$ are both bounded lattices, that is, with bottom and top elements.

In studies of bilattice, $\otimes k$ and $\bigoplus k$ are used to denote the meet and join operations that correspond to $\leq_{k}$, respectively, and $\otimes t$ and $\bigoplus t$ are used to denote the meet and join operations that correspond to $\leq_{t}$, respectively (see Fig. 1(b)). The partial order $\leq k$ is intended to represent the knowledge or information order and $\leq t$ is intended to represent the truth order. In other words, we can say that the knowledge order reports on how much information we have about a particular statement $p$, whereas the truth order reports on how confident we are that $p$ is true or false. Interpreting $x \leq_{t} y$, we simply thereby mean that $y$ is truer than $x$; in turn, we interpret $x \leq_{k} y$ as meaning that the evidence underlying $x$ is subsumed by the evidence underlying $y[34,35]$. 


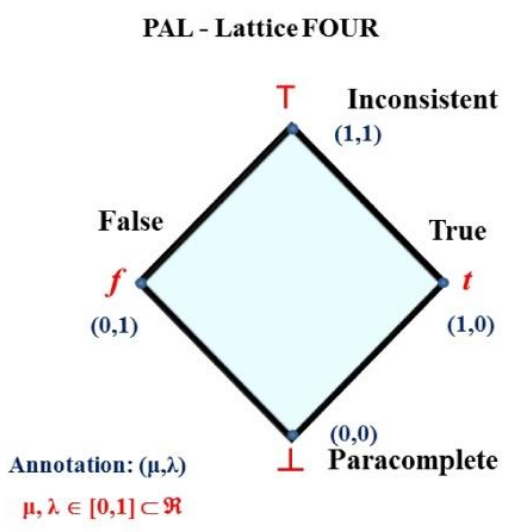

(a)

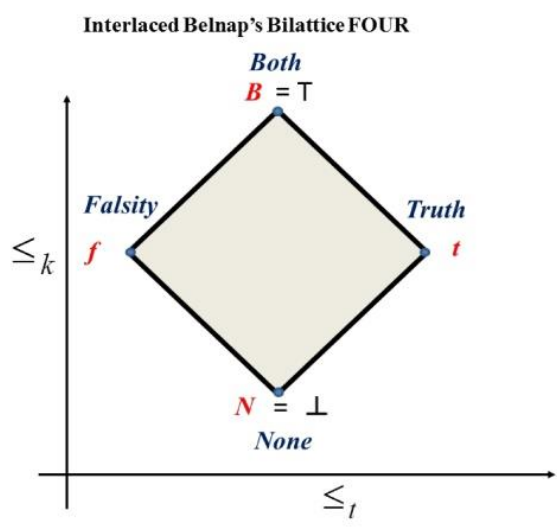

(b)

Fig. 1. (a) Lattice FOUR associated with PAL2v and representations of the extreme logical states in their vertices through the annotation $(\mu, \lambda)$. (b) Interlaced bilattice of Belnap with the ordering $\leq_{t}$ and $\leq_{k}$.

The normalized values in the PAL2v-interlaced bilattice FOUR enable mathematical analyses using data obtained in measurements made in the physical world. The paraconsistent equations are obtained from mathematical transformations that map the values arranged in a unitary square on the Cartesian plane (USCP) to the associated bilattice of PAL2v [27].

Initially, the degrees of evidence of PAL2v are considered on the USCP (which is also known as lattice $\kappa$ ) from where their values are mapped to lattice FOUR [32].

Given that, in the USCP, the values are allocated to the $x$-and $y$-axes, the mapping of the USCP (lattice $\kappa$ ) to the associated lattice $\tau$ of PAL2v is done by equating values with the degrees of evidence and implementing the following actions: (1) expansion of $\sqrt{2}$ from the $x$ - and $y$-axes; (2) counterclockwise rotation at the origin of $45^{\circ}$; and (3) translation of the -1 value from the $y$-axis.

These procedures are expressed as follows:

$$
\begin{aligned}
& T_{1}\left(X_{1}, Y_{1}\right)=(x \sqrt{2}, y \sqrt{2}) \quad(\text { expansion of } \sqrt{2}) ; \\
& T_{2}\left(X_{2}, Y_{2}\right)=\left(X_{1} \cdot \cos \theta-Y_{1} \cdot \sin \theta, X_{1} \cdot \sin \theta+Y_{1} \cos \theta\right) \quad\left(\text { counterclockwise rotation of } \theta=45^{\circ}\right) ; \\
& \cos \theta=\frac{1}{\sqrt{2}} \text { and } \sin \theta=\frac{1}{\sqrt{2}} ; \\
& T_{3}\left(X_{3}, Y_{3}\right)=\left(X_{2}, Y_{2}-1\right) \text { (translation of the }-1 \text { value from the } y \text {-axis); }
\end{aligned}
$$

Resulting to $T_{3}\left(X_{3}, Y_{3}\right)=(x-y, x+y-1)$.

If $x$ is the value allocated to the $x$-axis of the USCP and $y$ is the value allocated to the $y$-axis of the USCP, then $x=\mu$ and $y=\lambda$. The previously described actions create $T_{1}, T_{2}$, and $T_{3}$ transformations, as described in [33] and [36], that result in:

$$
T_{3}\left(X_{3}, Y_{3}\right)=(\mu-\lambda, \mu+\lambda-1)
$$

We denote the contradiction degree $(D c t)$ as $X_{3}$ and the certainty degree $(D c)$ as $Y_{3}$, as follows [33][36]: 
$X_{3}=D c_{(\mu, \lambda)} \rightarrow$ Certainty degree as a function of $\mu$ and $\lambda:$

$$
D c_{(\mu, \lambda)}=\mu-\lambda,
$$

$Y_{3}=\operatorname{Dct}_{(\mu, \lambda)} \rightarrow$ Contradiction degree as a function of $\mu$ and $\lambda:$

$$
D c t_{\mu, \lambda)}=\mu+\lambda-1 .
$$

Fig. 2 (a) shows the sequences followed to obtain the equations of the paraconsistent transformations and associated bilattice of PAL2v in the degrees of certainty and contradiction in the $x$-and $y$-axes.

The maximum values of the degrees of certainty are -1 at the vertex of the extreme logical state "false" $(f)$ and +1 at the vertex of the extreme logical state "true" $(t)$.

The maximum values of the degrees of contradiction are -1 at the vertex of the extreme logical state "paracomplete" $(\perp)$ and +1 at the vertex of the extreme logical state "inconsistent" (T) [27][30].

PAL2v, when applied to quantum mechanics, is called paraquantum logic $(\mathrm{PqL})$. In the interlaced bilattice of the $\mathrm{PqL}$, the values are represented by a universe of complex numbers, where the degree of contradiction lies in the imaginary axis and the degree of certainty lies in the real axis with the origin at the point equidistant from the vertices of the bilattice; therefore, the degrees of certainty and contradiction are both equal to 0 [31-33].

Paraconsistent logical state $\varepsilon_{\tau}$, which defines the paraquantum logical state [31], is considered the point of intersection between the degrees of certainty $\left(D c_{(\mu, \lambda)}\right)$ and contradiction $\left(D c t_{(\mu, \lambda)}\right)$ located in the bilattice FOUR or bilattice of the PqL. Therefore, the representation of the paraquantum logical state $\varepsilon_{\tau}$ can be expressed as follows [31-33]:

$$
\varepsilon_{\tau(\mu, \lambda)}=\left(D c_{(\mu, \lambda)}, D c t_{(\mu, \lambda)}\right) .
$$

Through mapping, the bilattice associated with $\mathrm{PqL}$ becomes a lattice of values, where the equations obtained create pairs of the values of $D c_{(\mu, \lambda)}$ and $D c_{(\mu, \lambda)}$ that define infinite internal points of intersection. Each internal point of intersection composed of a pair of values is a single paraquantum logical state $\varepsilon_{\tau(\mu, \lambda)}$. The equations obtained from the transformations enable the determination of the distance between the paraquantum logical state represented by the pair of inseparable values $\left(D c_{(\mu, \lambda)}, D c t_{(\mu, \lambda)}\right)$ and the extreme logical states represented by the vertices of the bilattice. Given that $D c_{(\mu, \lambda)}$ and $\operatorname{Dct}_{(\mu, \lambda)}$ are dependent on the values of $\mu$ and $\lambda$, the distance between the logical state resulting from $\varepsilon_{\tau(\mu, \lambda)}$ and one of the extreme logical states $t, f$, T, or $\perp$ represented by the vertices of the PqL bilattice is dependent on the values of $\mu$ and $\lambda$ considered in the physical world. If we know the paraquantum logical state $\varepsilon_{\tau}$ in any region of the PqL bilattice, then we can derive the values of the degrees of certainty $D c_{(\mu, \lambda)}$ and contradiction $D c t_{(\mu, \lambda)}$. The values of the degrees of evidence can be calculated using the following equations [31-33]:

$$
\mu_{(p)}=\frac{1}{2} D c_{(\mu, \lambda)}+\frac{1}{2} D c t_{(\mu, \lambda)}+\frac{1}{2}
$$




$$
\lambda_{(p)}=-\frac{1}{2} D c_{(\mu, \lambda)}+\frac{1}{2} D c t_{(\mu, \lambda)}+\frac{1}{2} .
$$

Non-commutation exists between the degrees of evidence of the $\mathrm{PqL}$ and is explained by the logical negation operation denoted by the symbol $\neg$. The change of position of the degrees of evidence in the annotation negates proposition $P$. Therefore, given proposition $P$, its logical negation $\neg P$ is represented by the exchange of the degrees of evidence in the annotation, as follows [31-33]:

$$
\neg(\mu, \lambda)=(\lambda, \mu)
$$

An interlaced bilattice [31][33] in addition to the negation operation expressed in Eq. (9) also enables the application of the complementation and conflation operations. The logical complementation operation in the PqL, denoted by the symbol $\lrcorner$, is the explicit complement to the unit of the degrees of evidence in the annotation. Given proposition $P$ and its complement $\downarrow P$, we can express the complementation operation as follows:

$$
\lrcorner(\mu, \lambda)=(1-\mu, 1-\lambda) .
$$

The logical conflation operation in the PqL, denoted by the symbol- - , is explained by the negation operation, followed by the complement to the unit of the degrees of evidence in the annotation [31]. Given proposition $P$ and its conflation $-P$, we can express the conflation operation as follows:

$$
-(\mu, \lambda)=(1-\lambda, 1-\mu)
$$

For a logical-mathematical study, the interlaced bilattice associated with $\mathrm{PqL}$ can be divided into four quadrants [31], where (a) in Quadrant I, the degrees of certainty and contradiction are positive (there is no operator action on the annotation $(\mu, \lambda)$ ); (b) in Quadrant II, the degree of certainty is negative and the degree of contradiction is positive (this is an action of the logical negation operator $\neg$ over the annotation $(\mu, \lambda)$ ); (c) in Quadrant III, the degrees of certainty and contradiction are negative (this is an action of the logical complementation operator $\downarrow$ over the annotation $(\mu, \lambda)$ ); and in Quadrant IV, the degree of certainty is positive and the degree of contradiction is negative (this is an action of the logical conflation operator - over the annotation $(\mu, \lambda)$ ).

With the negation, complementation, and conflation operations only over the values of the degrees of certainty and contradiction obtained in Quadrant I, the results of the degrees of certainty and contradiction are obtained in the three other quadrants of the interlaced bilattice of the PqL. Therefore, given that we detect the paraquantum logical state in Quadrant I with the corresponding values of $D c_{(\mu, \lambda)}$ and $D c t_{(\mu, \lambda)}$, the negation operation results in a negative degree of certainty and unchanged degree of contradiction. This results in another paraquantum logical state in Quadrant II, which is represented by $\left(-D c_{(\mu, \lambda)},+D c t(\mu, \lambda)\right)$ [31][33]. Similarly, the complementation operation on the values of the degrees of certainty and contradiction in Quadrant I results in negative values for both the degrees of certainty and contradiction. This result defines the paraquantum logical state in Quadrant III, which is represented by $\left(-D c_{(\mu, \lambda)},-D c t(\mu, \lambda)\right)$. The conflation operation on the values of the degrees of certainty and contradiction in Quadrant I results in positive values for the degree of certainty and negative values for the degree of contradiction. This result defines the paraquantum logical state in Quadrant IV, which is represented by $\left(+D c_{(\mu, \lambda)},-D c t(\mu, \lambda)\right)[31,32]$. 
Fig. 2(b) shows the interlaced bilattice of the PqL with quadrant operators and paraconsistent equations for reversible logic.

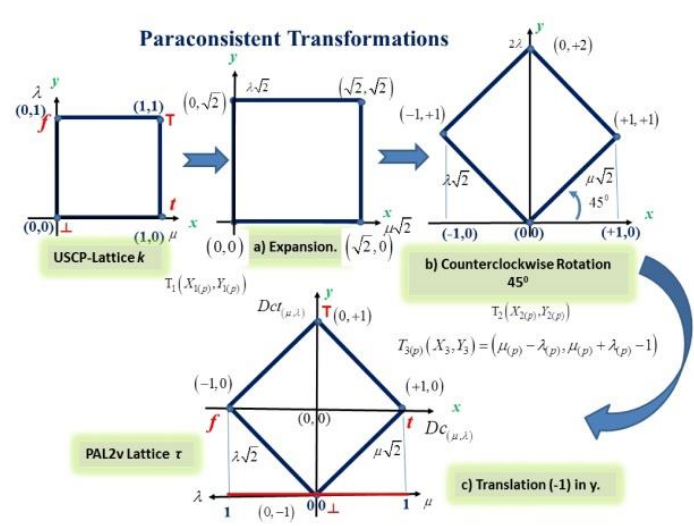

(a)

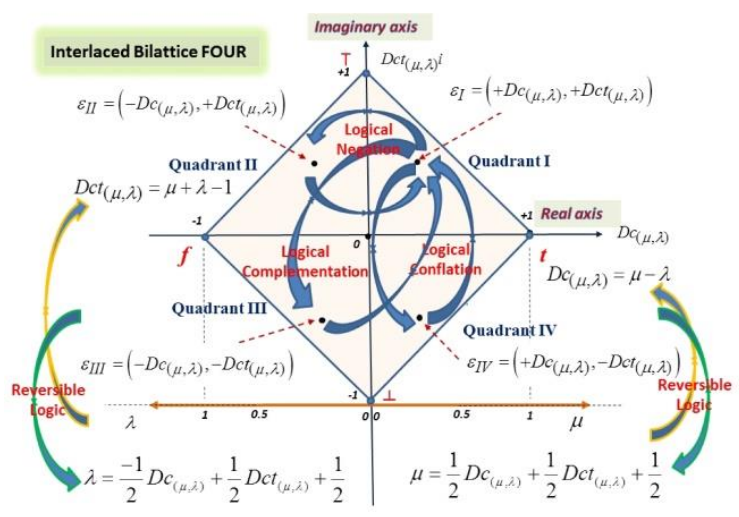

(b)

Fig. 2. (a) Sequences followed to obtain the equations of the paraconsistent transformations with counterclockwise rotation. (b) Interlaced bilattice of the PqL with quadrant operators and paraconsistent equations for reversible logic.

The objective of this work is to construct a paraconsistent model of the atom based entirely on PAL fundamentals so that the main concepts of quantum mechanics are satisfied and well equated to good computational practicality. The paraconsistent model of the atom will be presented and analyzed in sequence. In the first stage of the analysis, we show the trajectory of the logical states in the fundamental layer and its main equations obtained in Quadrant I of the interlaced bilattice of the PqL. Moreover, the negation, complementation and conflation operations will be applied and the model of the complete atom in the $x y$ plane will be formed in the perception of an observer in the vector base $X$. In the second stage of the analysis, the modeling equations of the complete atom will be presented and the trajectories of pure (nondegenerate) and impure (degenerate) quantum states will be highlighted. In the third stage of the analysis, the modeling equations of the energy layers are derived from the mapping of the degrees of evidence that differs in terms of the direction of rotation, which is now done clockwise. In this manner, the paraconsistent model of the atom in the $x y$ plane will be formed in the perception of an observer in the vector base $Y$. Finally, the results of an example of the application of the paraconsistent model of the atom will be presented with the energy values of the hydrogen atom. The results of the hydrogen atom show the curves obtained from the analysis of signals recorded by Raman spectroscopy using only Quadrant I of the interlaced bilattice of the PqL.

In the important work presented by Shannon in 1948 [36], the basis of the mathematical theory of communication or information theory was established. Moreover, Shannon's work emphasized a fundamental concept, that is, the entropy of the information, which became well known as the Shannon entropy.

The Shannon entropy has complementary interpretations that can be either information quantity (after measurement) or uncertainty (before measurement) in a given probability distribution. To establish the current concept that $H_{(\mathrm{s})}$ is a function of entropy comparable to Boltzmann's $H$ theorem, Shannon defined some statistical concepts through the equation $H_{(s)}=-k \sum_{i=1}^{n} p i \log p i$, where $p i$ is the probability of a system being in cell $i$ of its phase space and $k$ corresponds only to a certain unit of measure [37,38]. The equation of entropy in the case of two types of variables, that is, $p$ and $q=1-p$, is written as follows: 


$$
H_{(s)}=-k[p \log p+q \log q],
$$

where $p$ is the probability and $q$ is its complement $1-p$.

To obtain the maximum value of $H_{(s)}$ in Eq. (12), the unit value of $k$ is calculated using $k=\frac{\log 10}{\log 2} \simeq 3.321928$ [36][39].

The probability $p$ is an outcome that generates the degrees of evidence for the analysis of proposition $P$ for affirmation (true) or refutation (false). One form of representation whose results can be applied to the interlaced bilattice of the PqL is the Bernoulli trials process [31][38][40,41]. For this representation, we derive the random distribution of variable $X$, such that [31] $\operatorname{Pr}(X=1)=p$ and $\operatorname{Pr}(X=0)=q$. The expectation value is calculated using $\rightarrow E(X)=p$, and the variance of $X$, written as $\operatorname{Var}(X)$, is a measure of how much the value of $X$ varies from the expectation $E(X)$ [11]. The Bernoulli distribution is defined as follows:

$$
\operatorname{Var}\left(\mathrm{X} \nRightarrow p-p^{2}\right. \text {. }
$$

The standard deviation of the probability distribution is denoted by the symbol $\sigma$ and is defined as the square root of the variance $\operatorname{Var}(X)$, as follows:

$$
\sigma=\sqrt{\operatorname{Var}(X)}
$$

A graph of $\operatorname{Var}(X)$ as a function of $p \in[0,1]$ exhibits a parabola that opens downward [31][38].

In this work, we will present an analogy between the modes of use of probability in the Shannon entropy function and the Bernoulli distribution to determine the probability value $p$ of the paraconsistent analysis.

\section{Materials and Methods}

In the construction of the paraconsistent model of the atom, the concepts and equations of $\mathrm{PqL}$ and the logarithmic function of the Shannon entropy $H_{(\mathrm{s})}$ with the inclusion of the normalized Planck constant $\hbar$ are used. These fundamentals, equations, and concepts are applied to the in-depth analysis of the interlaced bilattice associated with PqL. In the proposed model, for the representation of the probabilistic functions according to the fundamentals of PqL, it was necessary to establish state vectors with unitary module that define the orbital paths and energy layers of the atom.

\subsection{State Vector P $\psi$ and Internal and External Orbit Trajectories of Paraquantum Logical States}

We will present below the representation of the paraquantum logical states related to the state vectors.

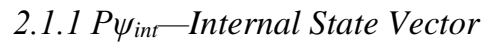

We consider an internal state vector with unitary module $\left(\mathrm{P} \psi_{\text {int }}\right)$ that has its origin located at the vertex of the true logical state $(t)$ of the interlaced bilattice of the PqL. Therefore, if the paraquantum logical state of the origin is expressed as $\varepsilon_{\tau}=(D c, D c t)=(+1,0)$, then the variation of the angle of inclination of the vector $(\mathrm{P} \psi$ int $)$ in Quadrant I will be $0 \leq \alpha_{\psi} \leq \frac{\pi}{4}$ in radians. Given that the modulus is unitary, the variation of the angle of inclination $\alpha_{\psi}$ of the vector $\mathrm{P} \psi_{\text {int }}$ will occur in the interlaced bilattice of the $\mathrm{PqL}$, with a curvilinear trajectory defined by the paraquantum logical states located at its end.

The internal trajectory in Quadrant I of the interlaced bilattice of the PqL was defined in Eq. (6). 


\subsubsection{P $\psi_{\text {ext }}$ External State Vector}

For an external state vector with unitary module $\left(\mathrm{P} \psi_{\mathrm{ext}}\right)$ that has its origin at the point equidistant from the vertices of the interlaced bilattice of the $\mathrm{PqL} \varepsilon_{\tau}=(D c, D c t)=(0,0)$, the equation of the paraquantum logical states that form the external trajectory in Quadrant I can be described as a function of the value of $\alpha_{\psi}$ and can be expressed as follows:

$$
\varepsilon_{\psi(\alpha \psi)} I=(1-\cos (\alpha \psi), \operatorname{sen}(\alpha \psi)) .
$$

This expression is similar to the following function:

$$
\varepsilon_{(\mu, \lambda)} I=\left(1-D c_{(\mu, \lambda)}, D c t_{(\mu, \lambda)}\right),
$$

where, in this work, $\mu$ and $\lambda$ are the degrees of evidence represented by the probabilistic function based on the Shannon entropy.

\subsubsection{P $\psi_{\text {Cext }}$-Complementary External State Vector}

The external trajectory of the paraquantum logical states in Quadrant I can be completed for the variation of the angle of inclination $\alpha_{\psi}$ of another vector, that is, $\mathrm{P} \psi_{\text {Cext }}$. With the same values of $\mu$ and $\lambda$, an external complementary vector $\left(\mathrm{P} \psi_{\text {CextII }}\right)$ with unitary module is created simultaneously, with its origin at the point $\varepsilon_{\tau}=(D c, D c t)=(0,0)$ and its angle of inclination having a variation of $\frac{\pi}{4} \leq \alpha_{\psi} \leq \frac{\pi}{2}$ in radians. With the same quantitative values of the degrees of evidence, this complementary operation is applied to the degrees of certainty and contradiction, generating the paraquantum logical state $\psi_{I 2(P q L) E 1}$. The generated paraquantum logical state establishes the orbital trajectory of the state vector $\mathrm{P} \psi_{\mathrm{I} 2}$, whose inclination presents a variation of $45^{\circ}$ to $90^{\circ}$, that is, the angular variation of $\frac{\pi}{4}$ to $\frac{\pi}{2}$ radians. The paraquantum logical state, which constructs the orbital trajectory at the end of the new complementary state vector with the completed action in the degrees of certainty and contradiction, will be represented by $\varepsilon_{(\mu, \lambda)} I I=\uparrow \varepsilon_{(\mu, \lambda)} I$ in the following expression:

$$
\varepsilon_{(\mu, \lambda)}^{I I}=\left((1-D c t(\mu, \lambda)), 1-\left(1-D c_{(\mu, \lambda)}\right)\right)
$$

In the PqL, $\mu$ and $\lambda$ are represented by probabilistic functions that must present results that have their values varying simultaneously in the corresponding intervals, that is, $0.5 \leq \mu_{(p)} \leq 1.0$ and $0.5 \leq \lambda_{(p)} \leq 1.0$, respectively.

Fig. 3 shows Quadrant I of the interlaced bilattice of the PqL with the state vector with unitary module, which, with the variation of the angle of inclination $\alpha_{\psi}$, establishes the internal trajectory of the paraquantum logical states. In same mode, the external state vectors $\mathrm{P} \psi_{\mathrm{extI}}$ and $\mathrm{P} \psi_{\text {CextI }}$ establish the complete external trajectory of Quadrant I.

\subsection{Representation of the Degrees of Evidence of PqL as Probabilistic Functions}

First, we consider that $\mu_{(p)}$ is a probabilistic function of $f_{(p)}=X$, such that $p \in \Re$ and it is contained in the closed interval [0,1]. Therefore, $p$ represents a probability value and the normalized values $X$ and $X^{\prime}$ should be adapted to the interlaced bilattice of the PqL. The two probability values must also be presented to form an 
annotation. We also consider that, as an initial condition, the two probabilistic sources 1 and 2 are out of phase at the angle $\Theta$, such that, in the amplitude variation of the probability value $p$, the probabilistic function of source 2 generates another function, that is, $\lambda_{(p)}=X^{\prime}$.

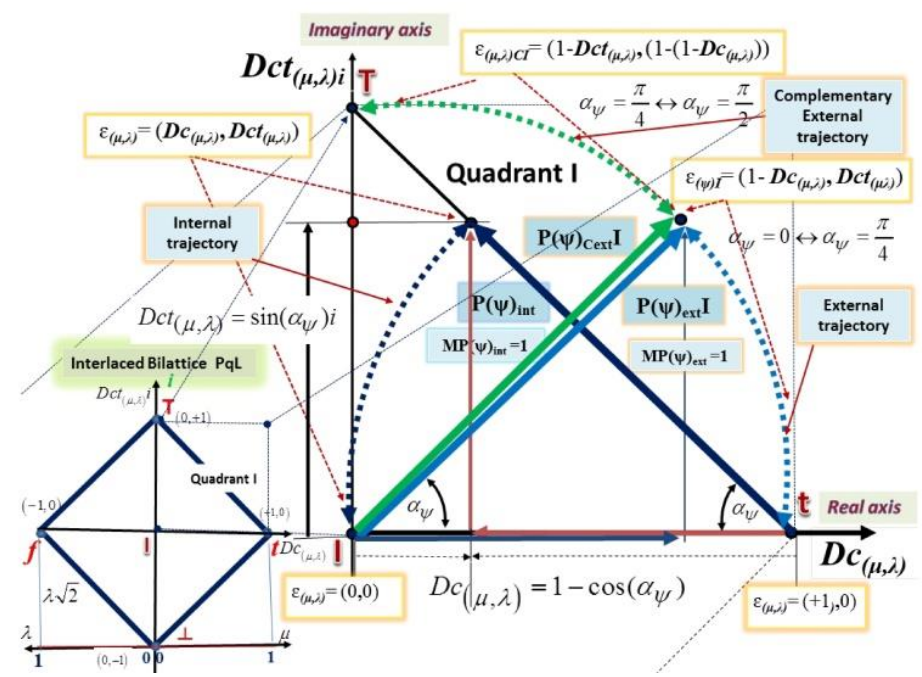

Fig. 3. Quadrant I of the interlaced bilattice of the $\mathrm{PqL}$, with the internal state vector $(\mathrm{P} \psi$ int $)$ and two external state vectors $\left(\mathrm{P} \psi_{\text {extI }}\right.$ and $\left.\mathrm{P} \psi_{\text {CextI }}\right)$ that establish the internal and external orbit trajectories of the paraquantum logical state $\varepsilon_{(\mu, \lambda)}$.

These two probabilistic functions must have the following characteristics: (a) when $X$ is at its maximum unitary value, that is, $\mu_{(p)}=X=1$, the difference between $X$ and $X^{\prime}$ will be equal to $\Delta_{(X p)}=\left(X-X^{\prime}\right) \rightarrow \Delta_{(X p)}=\left(1-\frac{1}{\sqrt{2}}\right)$; (b) when $X$ is at half its maximum value, that is, $\mu_{(p)}=X=\frac{1}{2}$, the difference between $X$ and $X^{\prime}$ will be null $\Delta_{(X p)}=X-X^{\prime}=\left(\frac{1}{2}-\frac{1}{2}\right)=0$. From the reference probability value at source 1 , which is considered a degree of favorable evidence $\mu_{(p)}$, the degree of unfavorable evidence $\lambda_{(p)}$ under the conditions previously mentioned is derived as follows:

$$
\lambda_{(p)}=\sqrt{\frac{\mu_{(p)}}{2}} .
$$

From Eq. (4), the degree of certainty of the interlaced bilattice of the PqL, which is probabilistic, can be calculated as follows:

$$
D c_{(p)}=\mu_{(p)}-\sqrt{\frac{\mu(p)}{2}} .
$$

In the same manner, the degree of contradiction shown in Eq. (5) is also a probabilistic function, which can be calculated as follows:

$$
\operatorname{Dct}_{(p)}=\mu_{(p)}+\sqrt{\frac{\mu_{(p)}}{2}}-1 .
$$

From Eq. (6), the paraquantum logical state $\psi_{\mathrm{PqL}}$ that appears in the interlaced bilattice of the PqL will be represented by two probabilistic functions, as follows: 


$$
\psi \tau_{(p)}=\left(1-D c_{(p)}, D c t(p)\right)
$$

In the representation of the functions, the paraquantum logical state $\psi \tau(p)$ will form a probabilistic trajectory into the interlaced bilattice of the PqL. Moreover, the paraquantum logical state $\psi_{\mathrm{PqL}}$ will form a probabilistic trajectory out of the interlaced bilattice of the $\mathrm{PqL}$, with the origin of the state vector at the point equidistant from the vertices, thereby located where $D c_{(p)}=0$ and $D c t_{(p)}=0$. In this case, the paraquantum logical state $\psi_{\mathrm{p}}$ that forms an external orbit trajectory will be constructed with two probabilistic functions, as follows:

$$
\psi_{(p)}=\left(1-D c_{(p)}, D c t(p)\right)
$$

where $D c_{(p)}$ is obtained using Eq. (19) and $D c t_{(p)}$ is obtained using Eq. (20).

\subsection{Representation of PqL Degrees of Evidence as Shannon Entropy Functions}

In this work, we will construct the paraconsistent model of the atom using the Shannon entropy to operate as probabilistic function representative of the degrees of evidence $(\mu, \lambda)$ and introduce the normalized Planck constant $\hbar$ in the interlaced bilattice of the PqL. In this manner, the degree of evidence will be a probabilistic function that represents the energy in the paraconsistent model of the atom.

As presented in [3] and [5], the Planck constant is $h=6.626070040 \times 10^{-34} \mathrm{~J} \cdot \mathrm{s}$ and its normalized value is calculated using $\hbar=\frac{h}{2 \pi}=\frac{6.626070040}{2 \pi} \times 10^{-34} \mathrm{~J} \cdot \mathrm{s}$, which results in $\hbar=1.054571800 \times 10^{-34} \mathrm{~J} \cdot \mathrm{s}$, where the energy value is in Joules ( $\mathrm{J}$ ) and the time is in seconds (s). From Eq. (12), the Shannon entropy [2] is represented by the probabilistic logarithmic function $H_{(s)}=-k[p \log p+q \log q]$ and the value of $k$ is a constant calculated using $k=\frac{\log 10}{\log 2} \simeq 3.321928$. Given that the constant $k$ of the Shannon entropy function depends on the variable used, we can consider the same relation of magnitudes and ensure the inclusion of the normalized Planck constant in the Shannon entropy. Thus, in the previously presented equation, the value of $k$ used to normalize the Shannon entropy function is related to the normalized Planck constant with $\pi$ factor. Given that $k$ and $\pi$ are constants, the constant $k$ of the logarithmic function that expresses the Shannon entropy will be represented by the multiplication of two physical constants with all of the values obtained by the energy calculations performed on the interlaced bilattice of the PqL multiplied by $10^{-34} \mathrm{~J} \cdot \mathrm{s}$. With this relation of constants, the quantum Shannon entropy is represented by $H_{(s) P q L}=-\hbar \pi[p \log p+q \log q]$ or

$$
H_{(s) P q L}=-\hbar \pi[p \log p+(1-p) \log (1-p)]
$$

With the inclusion of the normalized Planck constant in the Shannon entropy, the probabilistic function of the degree of favorable evidence can be expressed as follows: 


$$
\mu_{(P q L)}=H_{(s) P q L},
$$

where $H_{(s) P q L}$ is obtained using Eq. (23).

With the inclusion of the normalized Planck constant in the Shannon entropy, the probabilistic function of the degree of unfavorable evidence can be expressed as follows:

$$
\lambda_{(P q L)}=\sqrt{\frac{H_{(s) P q L}}{2}},
$$

where $H_{(s) P q L}$ is obtained using Eq. (23).

Thus, the degrees of certainty $D c_{(P q L)}$ and contradiction $D_{(P q L)}$ are representations of quantized probabilistic energy functions. At the level of the fundamental layer of the atom, the degrees of certainty and contradiction represent the energy values, which are obtained using the quantized probabilistic evidence degrees. From Eq. (19), the probabilistic certainty degree of the fundamental energy level $E_{1}$ can be calculated as follows:

$$
D c_{(P q L) E 1}=H_{(s) P q L}-\sqrt{\frac{H_{(s) P q L}}{2}} .
$$

From Eq. (20), the probabilistic contradiction degree of the fundamental energy level $E_{1}$ can be calculated as follows:

$$
\operatorname{Dct}_{(P q L) E 1}=H_{(s) P q L}+\sqrt{\frac{H_{(s) P q L}}{2}}-1 .
$$

The values of the degrees of certainty and contradiction considered in the set of complex numbers $\mathbb{C}$, with their quantized probabilistic functions, represent the energy of the atom. In the proposed paraconsistent model of the atom, the fundamental energy level $E_{1}$ is represented by the point of origin of the real and imaginary axes, which will be located at the point equidistant from the vertices of the interlaced bilattice of the PqL. In this representation, the paraconsistent logical state $\psi_{P q l}$ that defines the external orbital trajectory in the fundamental layer, representing the complex numbers in Quadrant I, is expressed as follows:

$$
\psi_{I 1(P q L) E 1}=\left(1-D c_{(P q L) E 1}, D c t_{(P q L) E 1^{i}}\right) .
$$

The probabilistic functions of the paraquantum logical state $\psi_{I(P q L) E 1}$ establish the fundamental energy level $E_{1}$ and the external orbital trajectory in the fundamental layer of the model of the atom at the end of the state vector $\mathrm{P} \psi_{\mathrm{I} 1}$ with unitary module, that is, $M \psi_{E 1}=\sqrt{\left(1-D c_{(P q L) E 1}\right)^{2}+(D c t(P q L) E 1)^{2}}$ or

$$
M \psi_{E 1}=\sqrt{\left(1-\left(H_{(s) P q L}-\sqrt{\frac{H_{(s) P q L}}{2}}\right)\right)^{2}+\left(H_{(s) P q L}+\sqrt{\frac{H_{(s) P q L}}{2}}-1\right)^{2}} \text {, }
$$

where $H_{(s) P q L}$ is the probabilistic Shannon entropy function with normalized Planck constant presented in Eq. 


\subsection{Analogies between Quantum Mechanics and Paraquantum Logic}

Some concepts of PqL can be compared with the concepts of quantum mechanics on the basis of the equations obtained in Quadrant I of the interlaced bilattice of the PqL. Therefore; in the quantum mechanics the quantum state is represented by $|\psi\rangle=\alpha|0\rangle+\beta|1\rangle$ and vector norm represented by $\||\psi\rangle \|=|\alpha|^{2}+|\beta|^{2}$. The same paraquantum logical state in quantum mechanics will be achieved, with the following relations of equality: $\alpha=\left(1-D c_{(P q L)}\right)$ and $\beta=D c t(P q L)$. The quantum state of the quantum mechanics in the PqL is represented by the following Dirac notation [7]:

$$
|\psi\rangle=\left(1-D c_{(P q L)}\right)|0\rangle+D c t_{(P q L)}|1\rangle .
$$

In general, for $n$ number of states related to $E n$ layers of energies:

$$
\left|\psi_{n}\right\rangle=\left(1-D c_{(P q L)} E n\right)|0\rangle+D c t_{(P q L) E n}|1\rangle .
$$

The representation of the degrees of certainty and contradiction and the fundamental energy level $E_{1}$ will be unitary $\left(E_{1}=1\right)$ and represented by:

$$
E_{1 T o t a l}=\left(1-\left(H_{(s) P q L}-\sqrt{\frac{H_{(s) P q L}}{2}}\right)\right)^{2}+\left(\left(H_{(s) P q L}+\sqrt{\frac{H_{(s) P q L}}{2}}\right)-1\right)^{2},
$$

where the potential energy of the fundamental layer is $E_{P 1}=\left(1-\left(H_{(s) P q L}-\sqrt{\frac{H_{(s) P q L}}{2}}\right)\right)^{2}$ and the kinetic energy of the fundamental layer is $E_{c 1}=\left(\left(H_{(s) P q L}+\sqrt{\frac{H_{(s) P q L}}{2}}\right)-1\right)^{2}$, where $H_{(s) P q L}$ is the probabilistic function presented in Eq. (23).

\subsection{PqL Energy Equations for the X Observer}

In Quadrant I of the interlaced bilattice of the PqL, the Shannon entropy functions simultaneously create the trajectories of the paraquantum logical states at the ends of two state vectors, thus establishing the fundamental energy level $E_{1}$ of the quantum state of the particle. The state vector P $\psi_{\text {CextI }}$ constructed with the complementary action, in relation to the original vector $\mathrm{P} \psi_{\text {extI }}$, has the same characteristics and differs only in terms of the angular variation. For the $X$ observer, as defined in the mapping shown in Fig. 3, the projections of the real values in the $x$-axis, which represent the potential energy, and the imaginary values in the $y$-axis, which represent the kinetic energy, vary proportionally, indicating the equilibrium of values against the inherent probabilistic uncertainties of quantum mechanics.

\subsubsection{Fundamental Layer of the Atom in the Paraconsistent Model}

The energies of the fundamental layer are represented by the equations of the $\mathrm{PqL}$, with the adapted function of the Shannon entropy having only the probability $p$ as variable.

Using the logical operations of negation, complementation, and conflation, as well as the fundamentals of PqL, we will now define the $n$ energy equations that form the $n$ layers of the paraconsistent model of the atom. 
Initially, through these operations, the fundamental energy equations of the three other quadrants of the interlaced bilattice of the $\mathrm{PqL}$ are obtained, thus forming the fundamental energy level $E_{1}$. The negation operator applied to the functions of the paraquantum logical states that mark the orbital trajectory of the particle in the fundamental energy layer of Quadrant I derives Quadrant II, as follows:

$$
\begin{gathered}
\neg \psi_{(I) 1}=\psi_{(I I) 1}=(-\alpha, \beta) \rightarrow \psi_{(I I) 1}=\left(D c_{(P q L) E n}-1, D c t,(P q L) E n\right), \\
\neg \psi_{(I) 2}=\psi_{(I I) 2}=(\beta-1, \alpha-1) \rightarrow \psi_{(I I) 2}=\left(D c t_{,(P q L) E n}-1,-D c_{(P q L) E n}\right) .
\end{gathered}
$$

In Quadrant III, the complementation operator applied to the functions of the paraquantum logical states that mark the orbital trajectory of the particle in the fundamental energy layer of Quadrant I derives the following expressions:

$$
\begin{gathered}
\downarrow \psi_{(I) 1}=\psi_{(I I I) 1}=(-\alpha,-\beta) \rightarrow \psi_{(I I I) 1}=\left(D c_{(P q L) E n}-1,-D c t,(P q L) E n\right), \\
\downarrow \psi_{(I) 2}=\psi_{(I I I) 2}=(\beta-1,1-\alpha) \rightarrow \psi_{(I I) 2}=\left(D c t_{(P q L) E n}-1,1-\left(1-D c_{(P q L) E n}\right)\right) .
\end{gathered}
$$

In Quadrant IV, the conflation operator applied to the functions of the paraquantum logical states that mark the orbital trajectory of the particle in the fundamental energy layer of Quadrant I derives the following expressions:

$$
\begin{aligned}
& -\psi_{(I) 1}=\psi_{(I V) 1}=(\alpha,-\beta) \rightarrow \psi_{(I V) 1}=\left(1-D c_{(P q L) E n},-D c t,(P q L) E n\right) \\
& -\psi_{(I) 2}=\psi_{(I V) 2}=(\beta, 1-\alpha) \rightarrow \psi_{(I V) 2}=\left(1-D c t_{(P q L) E n}, D c_{(P q L) E n}\right) .
\end{aligned}
$$

These $\mathrm{PqL}$ logical operations create the paraconsistent model of the atom, where the trajectory of the particle in the fundamental layer is a unit radius circle composed of the probabilistic functions of the degrees of certainty and contradiction. These trajectories related to fundamental energy are shown in the "Results" section.

\subsection{Energy Layers of Degenerate and Pure (or Nondegenerate) States}

In this work, we consider that the layers of the atom that relate to the degenerate states are represented by the energy that is related to the fundamentally pure state but is not aligned to the $x$-axis of the real values. In the interlaced bilattice of the $\mathrm{PqL}$, the degenerate states have different values of contradiction degrees, which bring them close to the extreme logical state of inconsistency in Quadrants I and II and the extreme logical state of paracompleteness in Quadrants III and IV.

In the same manner, the layers of the atom that relate to the pure or nondegenerate states are represented by the energy that is related to the fundamentally pure state. In the interlaced bilattice of the $\mathrm{PqL}$, the nondegenerate states are aligned to the $x$-axis of the real values, thus to the axis of the degrees of certainty. The nondegenerate states have the same values of contradiction degrees and different values of certainty degrees, which bring them close to the extreme logical state of true in Quadrants I and IV and the extreme logical state of false in Quadrants II and III.

\subsubsection{Second Layer of Energy}

With these considerations, for the second layer, the degree of favorable evidence $\mu$ is expressed in Eq. (24) and that of unfavorable evidence is expressed in Eq. (25). 
We can maintain a constant difference between the two degrees of evidence within a reasonable range of the probability variation $p$. For this, the degree of unfavorable evidence can be obtained by its multiplication with the degree of favorable evidence, such that $\lambda_{(P q L) E E 2}=\mu_{(P q L) E 2} \times \lambda_{(P q L) E 2}$ or

$$
\lambda_{(P q L) E E 2}=H_{(s) P q L} \sqrt{\frac{H_{(s) P q L}}{2}} .
$$

With these values of the degrees of evidence, the degree of certainty for the energy level $E_{2}$ will have a constant value over a reasonable range of probability variation $p$. Therefore, $D c_{(P q L) E 2}=\mu_{(P q L) E 2}-\lambda_{(P q L) E E 2}$ or

$$
D c_{(P q L) E 2}=H_{(s) P q L}-\left(H_{(s) P q L} \sqrt{\frac{H_{(s) P q L}}{2}}\right) .
$$

Moreover, the degree of contradiction for the energy level $E_{2}$ can be derived as follows:

$$
\operatorname{Dct}_{(P q L) E 2}=H_{(s) P q L}+\left(H_{(s) P q L} \sqrt{\frac{H_{(s) P q L}}{2}}\right)-1,
$$

where $H_{(s) P q L}$ is the probabilistic Shannon entropy function with normalized Planck constant presented in Eq.

In the second layer, the degenerate paraquantum logical state will be represented by the function:

$$
\psi_{(P q L) E} 2=\left(1-D c_{(P q L) E 2}, D c t(P q L) E 2\right)
$$

or by the Dirac notation for the $X$ observer:

$$
|\psi\rangle_{(P q L) E 2}=\left(1-D c_{(P q L) E 2}\right)|0\rangle+D c t(P q L) E 2|1\rangle
$$

where $D c_{(P q L) E 2}$ is presented in Eq. (34) and $D c t(P q L) E 2$ is presented in Eq. (35).

Given the relation to the pure state of the fundamental layer, in the second layer, the pure or nondegenerate paraquantum logical state will be represented by the complement expressed in Eq. (34) and the function expressed in Eq. (27), as follows:

$$
\psi_{(P q L) E \text { pure }}=\left(1-D c_{(P q L) E 2}, D c t_{(P q L) E 1}\right) .
$$

\subsubsection{Third Layer of Energy}

In the third layer, the degree of favorable evidence $\mu$ is equal to the degree of unfavorable evidence previously presented in Eq. (25). Therefore, $\mu_{(P q L) E 3}=\lambda_{(P q L) E 2}$ or

$$
\mu_{(P q L) E 3}=\sqrt{\frac{H_{(s) P q L}}{2}} .
$$

In this manner, the intermediary degree of unfavorable evidence will be obtained through the square root of $\mu_{(P q L) E 3}$. Therefore,

$$
\lambda_{(P q L) E 3}=\sqrt{\sqrt{\frac{H_{(s) P q L}}{2}}} .
$$

The degree of unfavorable evidence of the third layer will be obtained by its multiplication with the degree of favorable evidence, such that $\lambda_{(P q L) E E 3}=\mu_{(P q L) E 3} \times \lambda_{(P q L) E 3}$ or 


$$
\lambda_{(P q L) E E 3}=\sqrt{\frac{H_{(s) P q L}}{2}} \times \sqrt{\sqrt{\frac{H_{(s) P q L}}{2}}} .
$$

With these values of the degrees of evidence, the degree of certainty for the energy level $E_{3}$ will have a constant value over a reasonable range of probability variation $p$. Therefore, the degree of certainty for the third layer of energy will be computed using $D c_{(P q L) E 3}=\mu_{(P q L) E 3}-\lambda_{(P q L) E E 3}$ or

$$
D c_{(P q L) E 3}=\sqrt{\frac{H_{(s) P q L}}{2}}-\left(\sqrt{\frac{H_{(s) P q L}}{2}} \times \sqrt{\sqrt{\frac{H_{(s) P q L}}{2}}}\right) .
$$

Moreover, the degree of contradiction for the energy level $E_{3}$ can be derived as follows:

$$
\operatorname{Dct}_{(P q L) E 3}=\sqrt{\frac{H_{(s) P q L}}{2}}+\left(\sqrt{\frac{H_{(s) P q L}}{2}} \times \sqrt{\sqrt{\frac{H_{(s) P q L}}{2}}}\right)-1 .
$$

In the third layer, the degenerate paraquantum logical state will be represented by the function:

$$
\psi_{(P q L) E}=\left(1-D c_{(P q L) E 3}, D c t(P q L) E 3\right)
$$

or

$$
|\psi\rangle_{(P q L) E 3}=\left(1-D c_{(P q L) E 3}\right)|0\rangle+D c t(P q L) E 3|1\rangle,
$$

where $D c_{(P q L) E 3}$ is presented in Eq. (42) and $D c t_{(P q L) E 3}$ is presented in Eq. (43).

In the third layer, the pure or nondegenerate paraquantum logical state will be represented by the functions expressed in Eqs. (42) and (27), as follows:

$$
\psi_{(P q L) E 3 \text { pure }}=\left(1-D c_{(P q L) E 3}, D c t(P q L) E 1\right) .
$$

\subsubsection{Fourth Layer of Energy}

In the fourth layer, the degree of favorable evidence is equal to the degree of unfavorable evidence previously derived. Therefore, $\mu_{(P q L) E 4}=\lambda_{(P q L) E 3}$ or

$$
\mu_{(P q L) E 4}=\sqrt{\sqrt{\frac{H_{(s) P q L}}{2}}} .
$$

The degree of unfavorable evidence will be obtained through the square root of $\mu_{(P q L) E 4}$. Therefore,

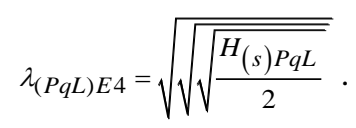

The degree of unfavorable evidence of the energy level $E_{4}$ of the current state is calculated by multiplication, such that $\lambda_{(P q L) E E 4}=\mu_{(P q L) E 4} \times \lambda_{(P q L) E 4}$ or

$$
\lambda_{(P q L) E E 4}=\sqrt{\sqrt{\frac{H_{(s) P q L}}{2}}} \times \sqrt{\sqrt{\sqrt{\frac{H_{(s) P q L}}{2}}}} .
$$

The degree of certainty for the energy level $E_{4}$ can be derived as follows: 


$$
D c_{(P q L) E 4}=\sqrt{\sqrt{\frac{H_{(s) P q L}}{2}}}-\sqrt{\sqrt{\frac{H_{(s) P q L}}{2}}} \times \sqrt{\sqrt{\sqrt{\frac{H_{(s) P q L}}{2}}}} .
$$

The degree of contradiction for the energy level $E_{4}$ can be derived as follows:

$$
D c t_{(P q L) E 4}=\sqrt{\sqrt{\frac{H_{(s) P q L}}{2}}}+\sqrt{\sqrt{\frac{H_{(s) P q L}}{2}}} \times \sqrt{\sqrt{\sqrt{\frac{H_{(s) P q L}}{2}}}}-1 .
$$

In the fourth layer, the degenerate paraquantum logical state will be represented by the function:

$$
\psi_{(P q L) E 4}=\left(1-D c_{(P q L) E 4}, D c t(P q L) E 4\right)
$$

or

$$
|\psi\rangle=\left(\left(1-D c_{n d(P q L) E 4}\right)\right)|0\rangle+\left(D c t_{n d(P q L) E 4}\right)|1\rangle
$$

where $D c_{(P q L) E 4}$ is presented in Eq. (50) and $D c t_{(P q L) E 4}$ is presented in Eq. (51).

In the fourth layer, the pure or nondegenerate paraquantum logical state will be represented by the functions expressed in Eqs. (50) and (27), as follows:

$$
\psi_{(P q L) E 3 \text { pure }}=\left(1-D c_{(P q L) E 4}, D c t(P q L) E 1\right)
$$

\subsubsection{Fifth Layer of Energy}

In the fifth layer, the degree of favorable evidence is equal to the degree of unfavorable evidence previously derived. Therefore, $\mu_{(P q L) E 5}=\lambda_{(P q L) E 4}$ or

$$
\mu_{(P q L) E 5}=\sqrt{\sqrt{\sqrt{\frac{H_{(s) P q L}}{2}}}} .
$$

The degree of unfavorable evidence will be obtained through the square root of $\mu_{(P q L) E 5}$. Therefore,

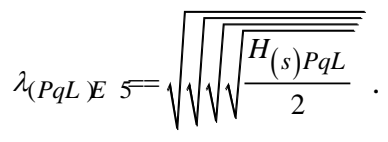

Given the relation to the pure state of the fundamental layer, the degree of unfavorable evidence of the energy level $E_{5}$ of the current state is calculated by multiplication, such that $\lambda_{(P q L) E E 5}=\mu_{(P q L) E 5} \times \lambda_{(P q L) E 5}$ or

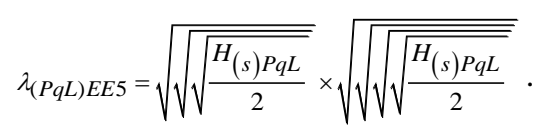

In this case, the degree of certainty for the energy level $E_{5}$ can be derived as follows:

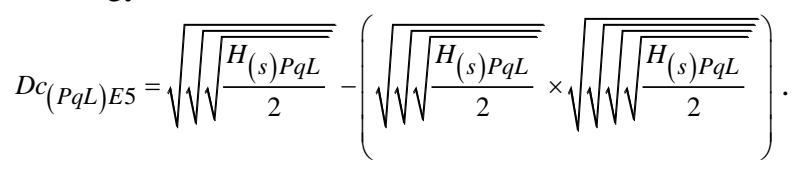

The degree of contradiction for the energy level $E_{5}$ can be derived as follows:

$$
D c t_{(P q L) E 5}=\sqrt{\sqrt{\sqrt{\frac{H_{(s) P q L}}{2}}}}+\left(\sqrt{\sqrt{\sqrt{\frac{H_{(s) P q L}}{2}}}} \times \sqrt{\sqrt{\sqrt{\sqrt{\frac{H_{(s) P q L}}{2}}}}}\right)-1 .
$$


In the fifth layer, the degenerate paraquantum logical state will be represented by the function:

$$
\psi_{(P q L) E 5}=\left(1-D c_{(P q L) E 5}, D c t(P q L) E 5\right)
$$

or

$$
|\psi\rangle=\left(\left(1-D c_{n d(P q L) E 5}\right)\right)|0\rangle+\left(D c t_{n d}(P q L) E 5\right)|1\rangle
$$

where $D c_{(P q L) E 5}$ is presented in Eq. (58) and $D c{ }_{(P q L) E 5}$ is presented in Eq. (59).

In the fifth layer, the pure or nondegenerate paraquantum logical state will be represented by the functions expressed Eqs. (58) and (27), as follows:

$$
\psi_{(P q L) E 5 \text { pure }}=\left(1-D c_{(P q L) E 5}, D c t_{(P q L) E 1}\right) .
$$

\subsubsection{Sixth Layer of Energy}

In the sixth layer, the degree of favorable evidence is equal to the degree of unfavorable evidence previously derived. Therefore, $\mu_{(P q L) E 6}=\lambda_{(P q L) E 5}$ or

$$
\mu_{(P q L) E 6}=\sqrt{\sqrt{\sqrt{\sqrt{\frac{H_{(s) P q L}}{2}}}}} .
$$

The degree of unfavorable evidence will be obtained through the square root of $\mu_{(P q L) E 6}$. Therefore,

$$
\lambda_{(P q L) E 6}=\sqrt{\sqrt{\sqrt{\sqrt{\sqrt{\frac{H_{(s) P q L}}{2}}}}}}
$$

The degree of unfavorable evidence of the energy level $E_{6}$ of the current state is calculated by multiplication, such that $\lambda_{(P q L) E E 6}=\mu_{(P q L) E 6} \times \lambda_{(P q L) E 6}$ or

$$
\lambda_{(P q L) E E 6}=\sqrt{\sqrt{\sqrt{\sqrt{\frac{H_{(s) P q L}}{2}}}}} \times \sqrt{\sqrt{\sqrt{\sqrt{\sqrt{\frac{H_{(s) P q L}}{2}}}}}} .
$$

The degree of certainty for the energy level $E_{6}$ can be derived as follows:

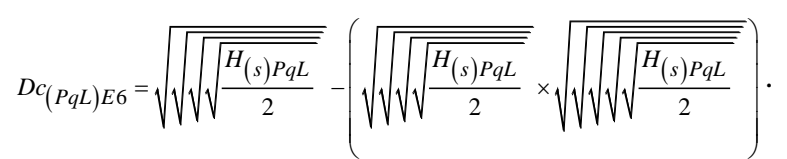

The degree of contradiction for the energy level $E_{6}$ can be derived as follows:

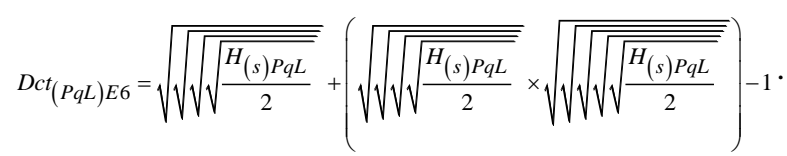

In the sixth layer, the degenerate paraquantum logical state will be represented by the function:

$$
\psi_{(P q L) E 6}=\left(1-D c_{(P q L) E 6}, D c_{(P q L) E 6}\right)
$$

or

$$
|\psi\rangle=\left(\left(1-D c_{(P q L) E 6}\right)\right)|0\rangle+\left(D c t_{(P q L) E 6}\right)|1\rangle
$$


where $D c_{(P q L) E 6}$ is presented in Eq. (66) and $D c t_{(P q L) E 6}$ is presented in Eq. (67).

In the sixth layer, the pure or nondegenerate paraquantum logical state will be represented by the functions expressed in Eqs. (66) and (27), as follows:

$$
\psi_{(P q L) E 6 \text { pure }}=\left(1-D c_{(P q L) E 6}, D c t_{(P q L) E 1}\right) .
$$

These procedures can be continued for $n$ layers of the paraconsistent model of the atom.

The graphs resulting from the energy layer equations are shown in the "Results" section.

\subsection{PqL Energy Equations for the Y Observer}

For the interlaced bilattice of the PqL, the equations that translate this situation can be obtained using the same procedures performed to obtain the equations that calculate the degrees of certainty and contradiction. To derive the equations for the $Y$ observer, we initially consider the same probabilistic function used for the $X$ observer, with its values allocated to the same USCP (lattice $k$ ). To obtain the degrees of certainty and contradiction for the $Y$ observer, we will apply the actions that previously created the transformations that resulted in the degrees of certainty and contradiction, now considering that the rotation of $45^{\circ}$ will be clockwise.

These actions are (1) expansion of $\sqrt{2}$ from the $x$ - and $y$-axes; (2) clockwise rotation at the origin of $45^{\circ}$; and

(3) translation of the -1 value from the $x$-axis.

These procedures are expressed as follows:

$$
\begin{aligned}
& T_{1}\left(X_{1}, Y_{1}\right)=(x \sqrt{2}, y \sqrt{2}) \quad(\text { expansion of } \sqrt{2}) ; \\
& T_{2}\left(X_{2}, Y_{2}\right)=\left(X_{1} \cdot \cos \theta+Y_{1} \cdot \sin \theta, X_{1} \cdot \sin \theta-Y_{1} \cos \theta\right) \quad\left(\text { clockwise rotation of } \theta=45^{\circ}\right) ; \\
& \cos \theta=\frac{1}{\sqrt{2}} \text { and } \sin \theta=\frac{1}{\sqrt{2}} ; \\
& T_{3}\left(X_{3}, Y_{3}\right)=\left(X_{2}-1, Y_{2}\right) \quad \text { (translation of the }-1 \text { from the } y \text {-axis); }
\end{aligned}
$$

Resulting to $T_{3}\left(X_{3}, Y_{3}\right)=(x+y-1, x-y)$ or

$$
T_{3(p)}\left(X_{3}, Y_{3}\right)=\left(\mu_{(p)}+\lambda_{(p)}-1, \mu_{(p)}-\lambda_{(p)}\right)
$$

We denote the contradiction degree $(D c t)$ as $X_{3}$ and the certainty degree $(D c)$ as $Y_{3}$, as follows: $X_{3}=D c \rightarrow$ Certainty degree:

$$
D c_{(p) y}=\mu_{(p)}+\lambda_{(p)}-1
$$

$Y_{3}=D c t \rightarrow$ Contradiction degree:

$$
\operatorname{Dct}_{(p) y}=\mu_{(p)}-\lambda_{(p)} .
$$

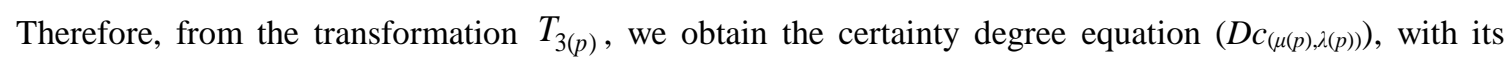
values projected on the $y$-axis of the lattice $\tau$, and the contradiction degree equation $\left(\operatorname{Dct}_{(\mu(p), \lambda(p))) \text {, with its values }}\right.$ projected on the $x$-axis of the lattice $\tau$. 
In Fig. 4(a), the sequences of the paraconsistent transformations for the $Y$ observer are shown.

Given that the paraquantum logical state will have its values changed through the modification of the orbital trajectory, for the $Y$ observer, its representation will be at the extremity of the internal state vector of the PqL, originating from the vertex where the inconsistent logical state is located. For logical negation, the internal state vector will have its origin at the vertex, where the extreme logical state paracomplete is located.

Given that the paraquantum logical state $\psi \tau$ is the point of intersection between the degree of certainty $(D c)$ and the degree of contradiction $(D c t)$ located in the interlaced bilattice of the $\mathrm{PqL}$, the representation of the $Y$ observer in the form of a set of complex numbers will be expressed as follows [18][22,23]:

$$
\psi_{\psi \text { int }} I y=i D c_{(p)}+D c t_{(p)} .
$$

An internal state vector with unitary module that originates from the inconsistent extreme logical state (T) is derived as follows:

$$
M \psi y=\sqrt{\left(D c_{(p)}\right)^{2}+\left(D c t_{(p)}\right)^{2}} .
$$

This internal state vector with unitary module defines the orbital paths within the interlaced bilattice of the PqL. The same values represent the external logical state for the fundamental energy layer, which can be expressed as follows:

$$
\psi_{\psi \mathrm{int}} I y=i\left(1-D c_{(p) y}\right)+D c t_{(p) y} .
$$

This unitary module vector defines the external orbital trajectory in the bilattice of the PqL. The external orbital trajectory is made at the extremity of the external state vector with unitary module whose origin is at the point equidistant from the vertices of the interlaced bilattice of the PqL. Its module is expressed as follows:

$$
M \psi I y=\sqrt{\left(1-D c_{(p) y}\right)^{2}+\left(D c t_{(p) y}\right)^{2}} .
$$

The values representing the external logical state of the fundamental energy layer for the $Y$ observer with the probability values used in the PqL can be derived as follows:

$$
\psi_{\psi y}=\left(D c_{(p) y}, D c t_{(p) y}\right) \text {. }
$$

Fig. 4(b) shows Quadrant I of the interlaced bilattice of the PqL with the state vectors and orbit trajectories of the $Y$ observer.

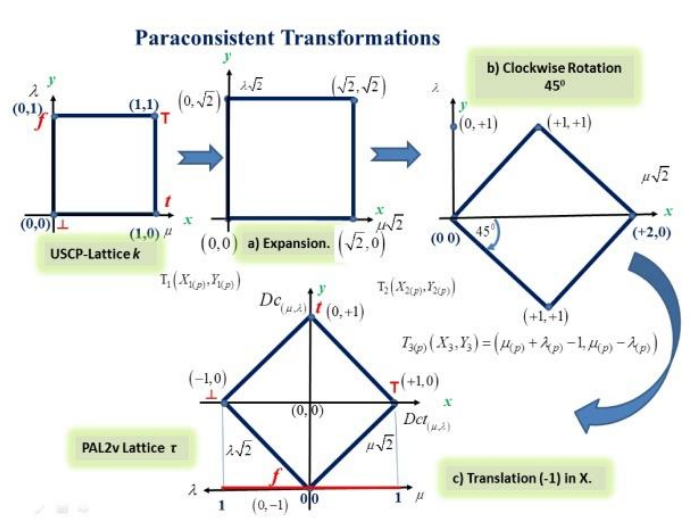

(a)

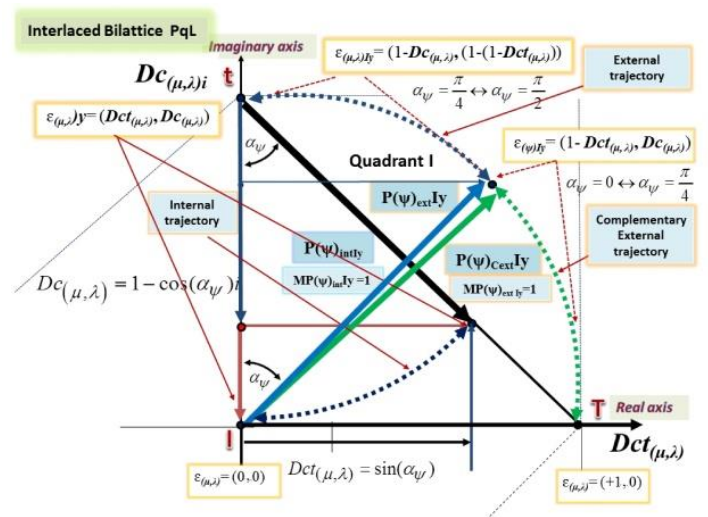

(b) 
Fig. 4. (a) Sequences of the paraconsistent transformations for the $Y$ observer with clockwise rotation. (b)

Quadrant I of the interlaced bilattice of the PqL with the vectors and orbit trajectories of the $Y$ observer.

\subsection{A Representation of the Paraconsistent Model of the Atom}

The mapping sequences of probabilistic evidence degrees with both the $X$ and $Y$ observers result in equations of superposed paraquantum logical states in Quadrant I of the interlaced bilattice of the PqL. Using the paraquantum equations, we can present the results of the superposed logical states as two bilattices comprising one superposed plane.

\subsubsection{Superposed Paraconsistent Logical States}

The energy equations for the $Y$ observer are represented by the quantum logical states with a set of complex numbers, where the imaginary and real values will change depending on the observer. For the $Y$ observer, the vector base will be orthogonal to the base $X$. This means that, for the $Y$ observer, the imaginary values of the $X$ observer will be their real values and the actual values of the $X$ observer will be their imaginary values.

Fig. 5(a) shows the sequences of the paraconsistent transformations for the $X$ and $Y$ observers and framework vectors.

Fig. 5(b) shows the interlaced bilattice of the $\mathrm{PqL}$ and the vectors and trajectories of the $X$ and $Y$ observers with superposed paraquantum logical states.

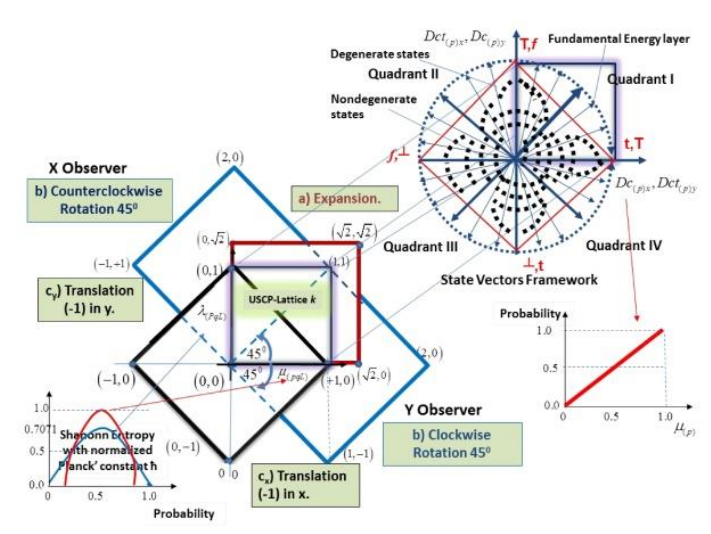

(a)

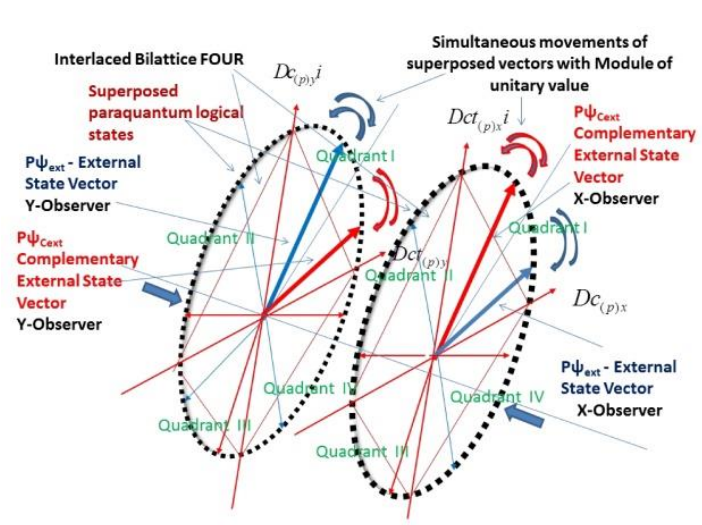

(b)

Fig. 5. (a) Sequences of the paraconsistent transformations for the $X$ and $Y$ observers and framework vectors. (b) Interlaced bilattices with the vectors and orbit trajectories of the $X$ and $Y$ observers with superposed paraquantum logical states.

In the next section, a paraconsistent model of the atom will be constructed with the equations of the Shannon entropy functions and the normalized Planck constant. In the paraconsistent model, the equations form the superposed paraquantum logical states located in the planes of the $X$ and $Y$ observers. Moreover, variations of the probability values will be applied to the equations expressing the orbital trajectories of the particles in the two superposed planes as traces of energy in the overlapping layers of the atom.

\section{Results}

The results that will be presented are from simulations using the paraconsistent equations of the model of the atom in a calculi spreadsheet. In the simulations using the energy equations, the probability values vary with 
intervals in the order of $1 / 100$, thus generating the favorable and unfavorable degrees of evidence through the Shannon function with the inclusion of the normalized Planck constant $\hbar$.

The normalized Planck constant $\hbar$ value used was $\hbar=1.0545718$, and the Pi constant value used was $\pi=3.141592654$.

\subsection{Results related to the Fundamental Equations of the Paraconsistent Model of the Atom}

Fig. 6(a) shows the graphs of the results of the degree of favorable evidence $\mu_{(\mathrm{PqL})}$ derived using Eq. (24) and the degree of unfavorable evidence $\lambda_{(\mathrm{PqL})}$ derived using Eq. (25) after the application of the Shannon entropy function with the inclusion of the normalized Planck constant $\hbar$. Fig. 6 (b) shows the variations of the degrees of certainty (Eq. (26)) and contradiction (Eq. (27)) and the simulation results of the unitary module $M(\psi) \mathrm{I}_{1}$ of the quantized probabilistic function (Eq. (29)) for a complete variation of probability $p$.

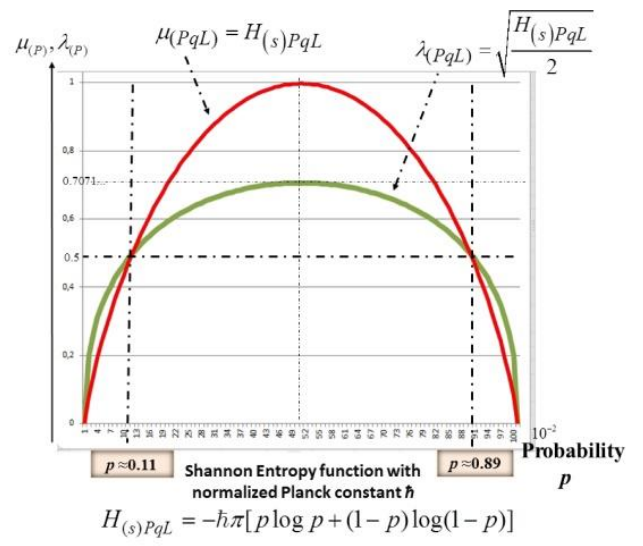

(a)

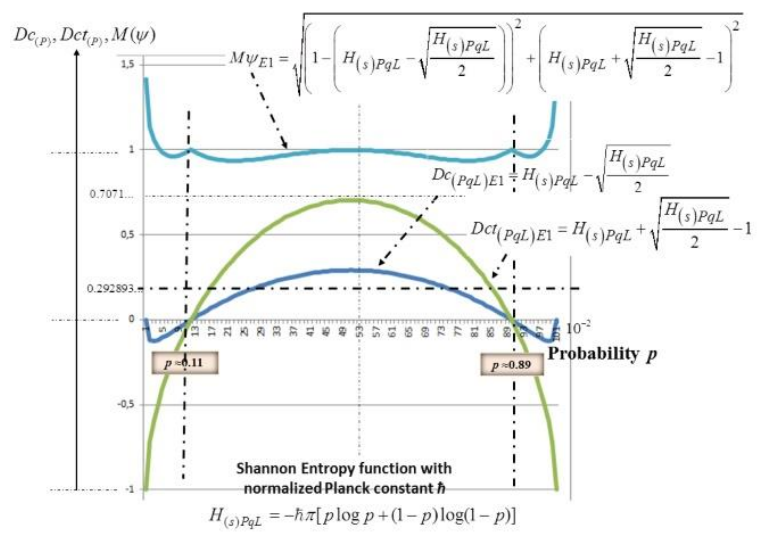

(b)

Fig. 6. (a) Graphs of the results of the degrees of favorable evidence $\mu_{\text {(p) }}$ (Eq. (24)) and unfavorable evidence $\lambda_{\text {(p) }}$ (Eq. (25) after the application of the Shannon entropy function with the inclusion of the normalized Planck constant. (b) Simulation results of the degrees of certainty and contradiction, according to Eqs. (26) and (27), respectively, and the unitary module $\mathrm{M}(\psi) \mathrm{I}_{1}$ of the quantized probabilistic function (Eq. (29)) for a complete variation of probability $p$.

\subsection{Results related to the Energy Layers of the Paraconsistent Model of the Atom}

Fig. 7(a) shows the simulation results of the fundamental energy layer for the paraconsistent model of the atom. Fig. 7(b) shows the simulation results with explications about the utilized equations and the interlaced bilattice of the PqL circumscribed in the external orbital circumference. In this simulation, we used Eqs. (24) to (27) and the logical operations of negation, complementation, and conflation. 


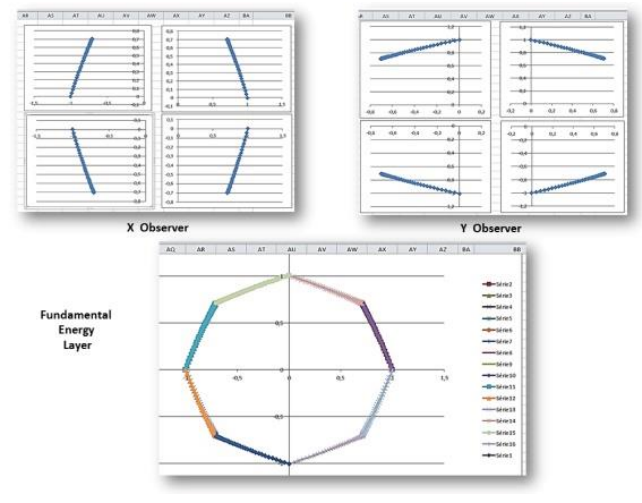

(a)

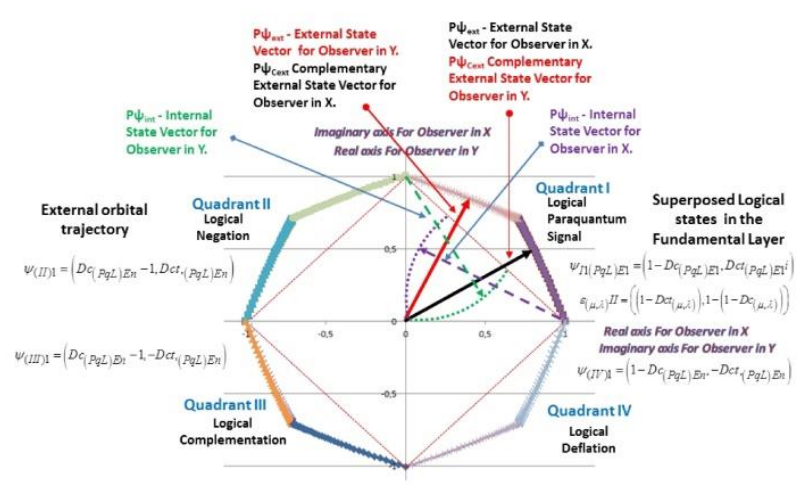

(b)

Fig. 7. (a) Simulation of the fundamental energy layer of an atom using the equations of Quadrant I and the PqL operators. (b) Simulation results with explications about the utilized equations and the interlaced bilattice of the

PqL circumscribed in the external orbital circumference.

Fig. 8(a) shows the results simulated with a group of Shannon entropy functions, which are obtained using the equations of the degrees of certainty in six layers of the paraconsistent model of the atom using the method to find the values for the pure or nondegenerate states. The results of the simulations obtained using the equations defining the six layers of the paraconsistent model of the atom for the degenerate state mode from the $X$ observer are shown in Fig. 8(b).

Fig. 8(c) shows the representation of the bilattice of the PqL, including the results for the degenerate state mode in the paraconsistent model of the atom from the $X$ observer. In this simulation, we used Eqs. (24) to (27), (33) to (35), (39) to (43), (47) to (51), (55) to (59), and (63) to (67) and the logical operations of negation, complementation, and conflation.

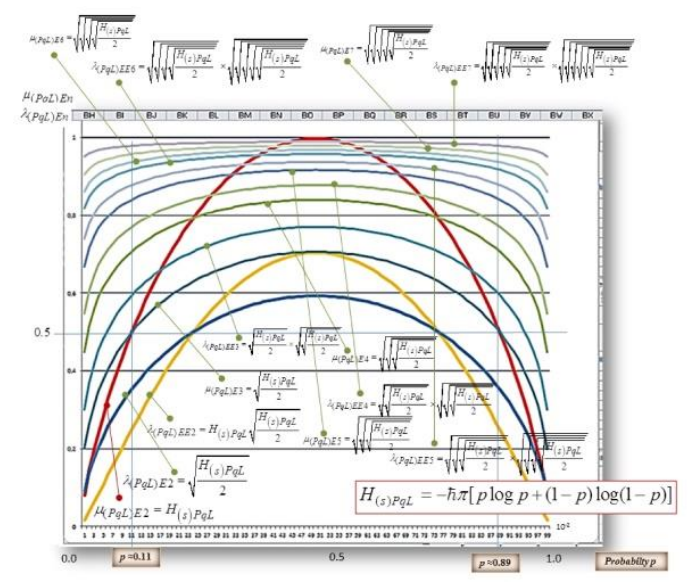

(a)

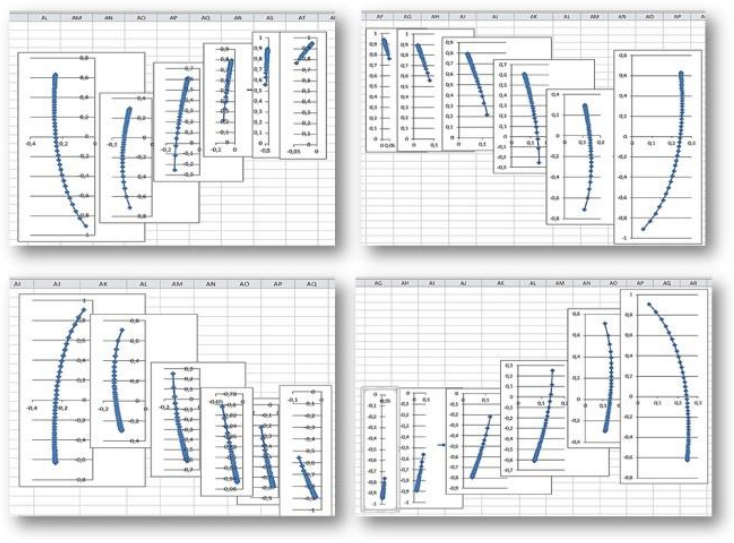

(b) 


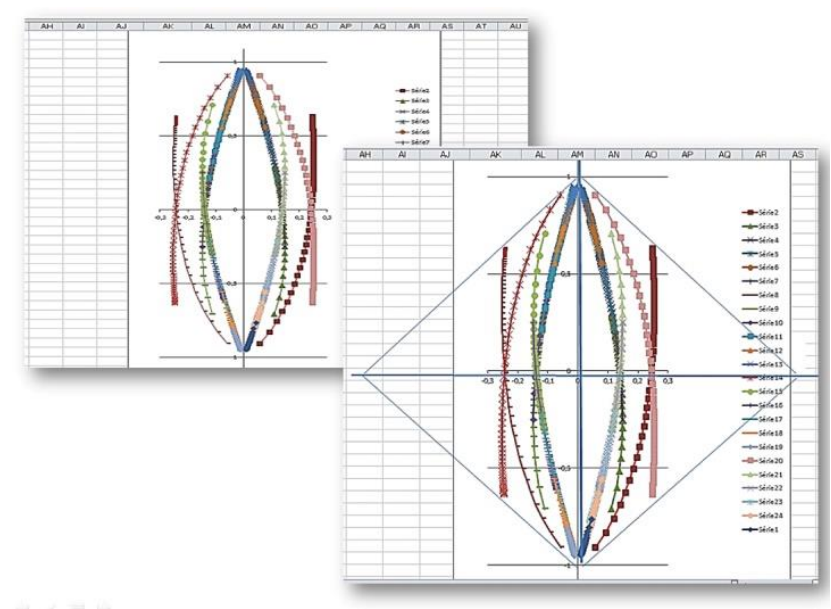

(c)

Fig. 8. (a) Simulation results obtained using a group of Shannon entropy functions for calculating the certainty degree equations in six layers of the paraconsistent model of the atom for the pure or nondegenerate states. (b) Simulation of the energy layer with degenerate states of an atom using the paraquantum equations in mode from the $X$ observer. (c) Representation of the bilattice of the PqL, including the results for the degenerate state mode in the paraconsistent model of the atom from the $X$ observer.

The simulation results obtained using the equations defining the six layers of the paraconsistent model of the atom for the degenerate state mode from the $Y$ observer are shown in Fig. 9(a). Fig. 9(b) shows the representation of the simulation of the degenerate states of an atom in mode from the $Y$ observer with the inclusion of the bilattice of the PqL. In this simulation, we used the adapted equations shown in the "PqL Energy Equations for the $Y$ Observer" section, with the logical operations of negation, complementation and conflation.

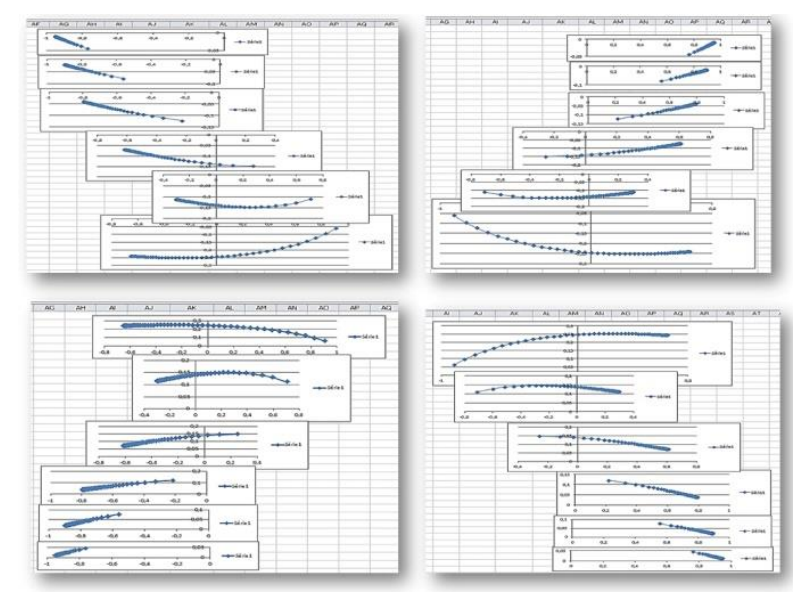

(a)

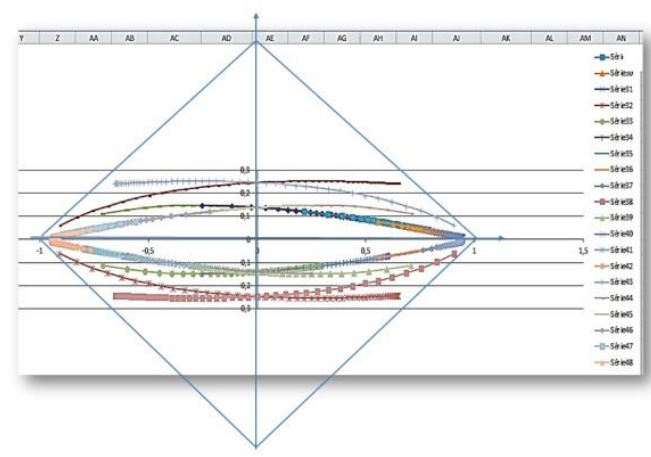

(b)

Fig. 9. (a) Simulation of the energy layers with degenerate and nondegenerate states of an atom using the paraquantum equations in mode from the $Y$ observer. (b) Representation of the simulation of the degenerate states of an atom in mode from the $Y$ observer with the inclusion of the bilattice of the PqL. 
Fig. 10(a) shows the results of the simulation of a complete paraconsistent model of the atom without nondegenerate states. Fig. 10(b) shows the simulation results with explications about the utilized equations and the interlaced bilattice of the PqL circumscribed in the orbital circumference and energy levels. Fig. 10(c) shows the paraconsistent model of the complete atom with the orbital energy paths of the layers represented by the degrees of certainty and contradiction for the two references of the $X$ and $Y$ observers. In this simulation, we used Eqs. (24) to (76) and the adapted equations shown in the "PqL Energy Equations for the $Y$ Observer" section, with the logical operations of negation, complementation, and conflation.

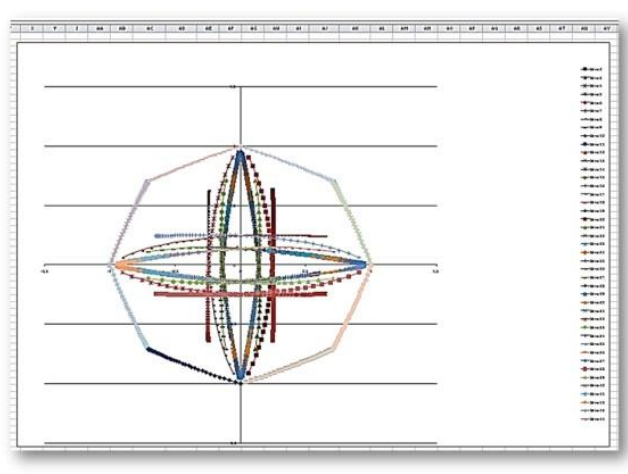

(a)

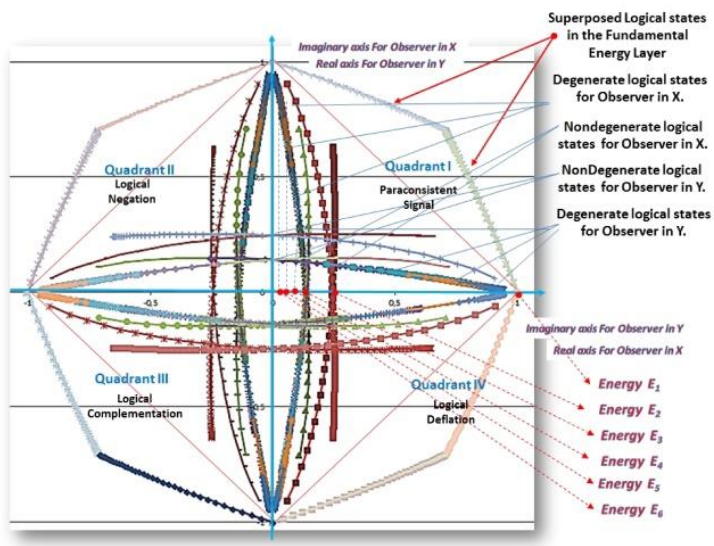

(b)

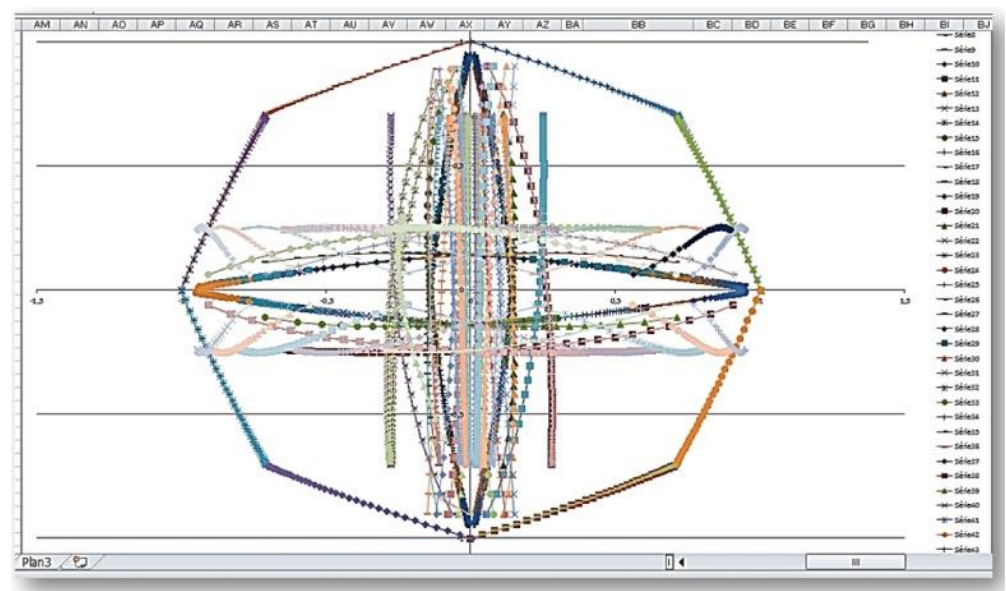

(c)

Fig. 10. (a) Simulation results of the paraconsistent model of the atom without nondegenerate states, with the representation of the interlaced bilattice of the PqL. (b) Simulation results of the complete paraconsistent model of the atom with degenerate and nondegenerate states. (c) Simulation results of the complete paraconsistent model of the atom.

\subsection{Results related to the Wave Functions in the Paraconsistent Model of the Atom}

With the probabilistic variables established by the Shannon entropy functions in which the normalized Planck constant $\hbar$ is included, the values of $D c_{(P q L)}$ and $D c t_{(P q L)}$ are transformed into functions representing the energies emanating from the logical model of the atom. The wave function for each layer of the 
paraconsistent model of the atom is derived by multiplication of the paraquantum logical state with the conjugate complex, according to Eq. (2). For the fundamental energy, the wave function $|\psi|^{2}$ is calculated using $\left|\psi_{\left(\mu_{(P q L)}, \lambda_{(P q L)}\right)}\right|^{2}=\psi(P q L) E 1 \times \psi^{*}(P q L) E 1$ or

$$
\left.\mid \psi_{\left(\mu_{(P q L}\right)}, \lambda_{(P q L)}\right)\left.\right|^{2}=\left[\left(1-D c_{(P q L) E 1}\right)+D c t_{(P q L) E 1^{i}} i \times\left[\left(1-D c_{(P q L) E 1}\right)-D c t_{(P q L) E 1^{i}}\right],\right.
$$

where $D c_{(P q L) E 1}$ is presented in Eq. (26) and $D c_{(P q L) E 1}$ is presented in Eq. (27).

For the other energy layers, the axes of the references and the amplitudes of the degrees of certainty and contradiction derived by the equations will be considered.

The graphs obtained by the applications of the equations that are related to the wave functions are shown in Fig. 11(a).

Fig. 11(b) shows the six wave functions represented in the paraconsistent model of the atom with orbital energy paths of layers $E_{1}, E_{2}$, and $E_{3}$ for the $X$ and $Y$ observers.

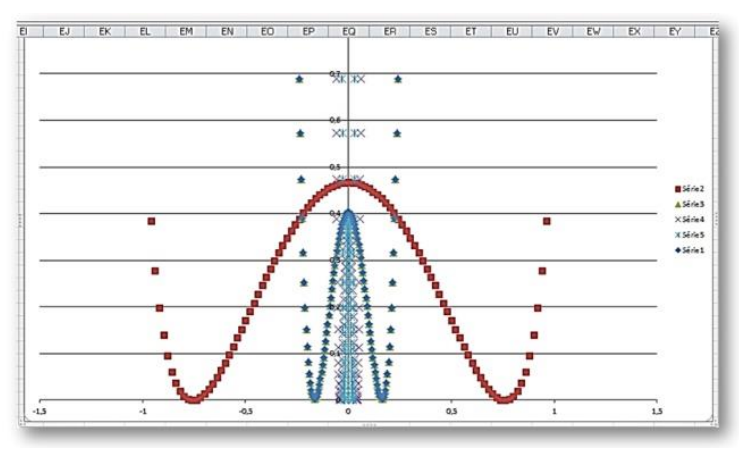

(a)

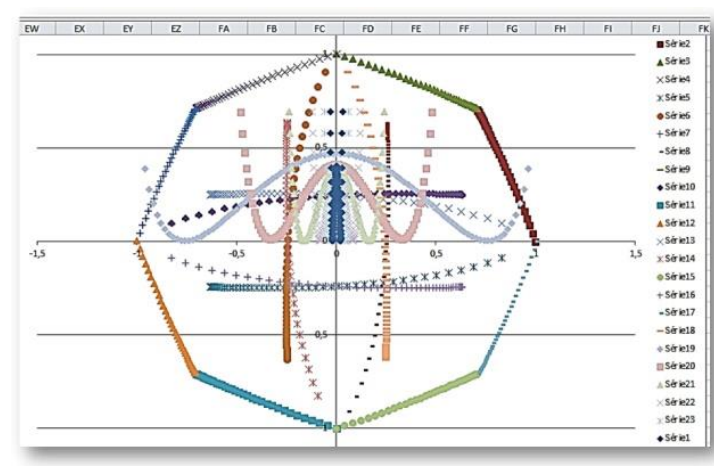

(b)

Fig. 11. (a) Simulation results of the wave functions for the fundamental energy layer and the wave functions for some energy layers. (b) Wave functions represented in the paraconsistent model of the atom with orbital energy paths of layers $E_{1}, E_{2}$, and $E_{3}$ for the $X$ and $Y$ observers.

\subsection{Results related to the Heisenberg Uncertainty Principle in the PqL}

The reversibility characteristic of the $\mathrm{PqL}$ ensures that the degrees of evidence of probability can be obtained through Eqs. (7) and (8). The certainty degrees at the $x$-axis $\left(D c_{(P q L) E}\right)$ and the contradiction degrees at the $y$-axis $\left(D_{\left(t_{(} q L\right) E}\right)$ in its associated interlaced bilattice can be mapped back to the USCP, where the degrees of evidence $\mu_{(\mathrm{p})}$ and $\lambda_{(\mathrm{p})}$ are plotted in the $x$ - and $y$-axes. For the typical PqL logical state, that is, $\psi_{(P q L)}=\left(D c_{(P q L)}, D c t(P q L)\right)$, the degree of favorable evidence when the value of the degree of contradiction is 0 can be calculated using $\mu_{(p)}=\frac{1}{2} D c_{(\mu, \lambda)}+\frac{1}{2}$, which is the normalized value for $D c_{(P q L) E}$. With this procedure, the 
normalized value for the contradiction degree is obtained using $\mu_{(p)}=\frac{1}{2} D c t_{(\mu, \lambda)}+\frac{1}{2}$. We can make this normalization for the external PqL logical state of the type $\psi_{(P q L)}=\left(1-D c_{(P q L)}, D c t(P q L)\right)$, where the normalized value for $1-D c_{(P q L)}$ is considered the uncertainty for the $x$-axis computed as:

$$
\Delta x_{(P q L)}=\frac{\left(1-D c_{(P q L)}\right)+1}{2}
$$

and the normalized value for $\operatorname{Dct}_{(P q L)}$ is considered the uncertainty for the $y$-axis computed as:

$$
\Delta y_{(P q L)}=\frac{D c t_{(P q L)}+1}{2} .
$$

With Eqs. (80) and (81), the formal inequality of the Heisenberg uncertainty principle expressed in Eq. (1) is represented in the $\mathrm{PqL}$ as follows:

$$
\Delta x_{(P q L)} \Delta y_{(P q L)} \geq \frac{\hbar}{2}
$$

The graphical results of the simulations with the Heisenberg uncertainty principle equations are shown in Fig. 12(a).

\subsection{Results related to the Calculation of the Probability Value in the Paraconsistent Model of the Atom}

The $\mathrm{PqL}$ is a reversible logic, and in this manner, we can analyze the paraconsistent model of the atom from its inner part, considering the nucleus as the energy generator that spreads its values to its external part. With the equations considered in this manner, we can estimate the probable values of energy, as well as the probable location of the energy around the generating nucleus. The reversibility characteristic of the PqL ensures that the degrees of evidence of probability can be obtained through Eqs. (7) and (8) and the result of its normalized value can be compared with Bernoulli's probabilistic function presented in Eq. (13). This comparison shows that the following equality is a good approximation: $\mu_{(p)}=2 \sigma$, where $\sigma$ is the standard deviation of probabilistic measures. With $\sigma=\frac{\mu_{(p)}}{2}$ and variance $\operatorname{Var}=\sigma^{2}$, the variance in the PqL can be defined as $\operatorname{Var}=\left(\frac{\mu_{(p)}}{2}\right)^{2}=\frac{\mu_{(p)}}{4}$. The variance related to the degree of unfavorable evidence can be computed using $\operatorname{Var}=\lambda_{(p)}{ }^{4}$. In this case, the variance can be represented by the values of the degrees of certainty and contradiction, such that:

$$
\operatorname{Var}_{(P q L)}=\left(\frac{-D c_{(P q L)}+D c t(P q L)}{2}\right)^{4} \text {. }
$$

From Eq. (12), we can obtain the $p$ values by combining the variance equation with a second-degree equation and applying Bhaskara's method, such that:

$$
p=\frac{1+\sqrt{1-\left[4 \operatorname{Var}_{(P q L)}\right]}}{2} .
$$

For the complement, $q=1-p$ or 


$$
q=\frac{1-\sqrt{1-\left[4 \operatorname{Var}_{(P q L)}\right]}}{2} .
$$

Using Eqs. (80), (81), and (82), Fig. 12(a) shows the results of the simulation of the Heisenberg uncertainty principle. Fig. 12(b) shows the results of the simulation of Eqs. (84) and (85) with probability values ranging from 0 to 1.0 as $p$ and from 1.0 to 0 as complement $q=1-p$.

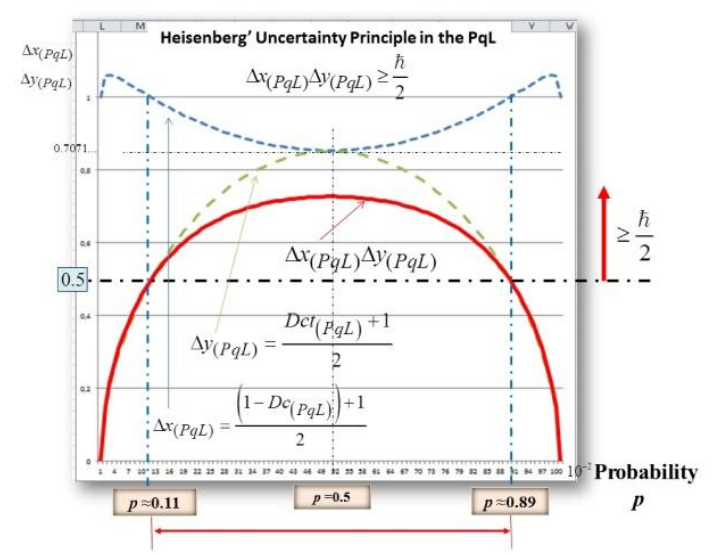

(a)

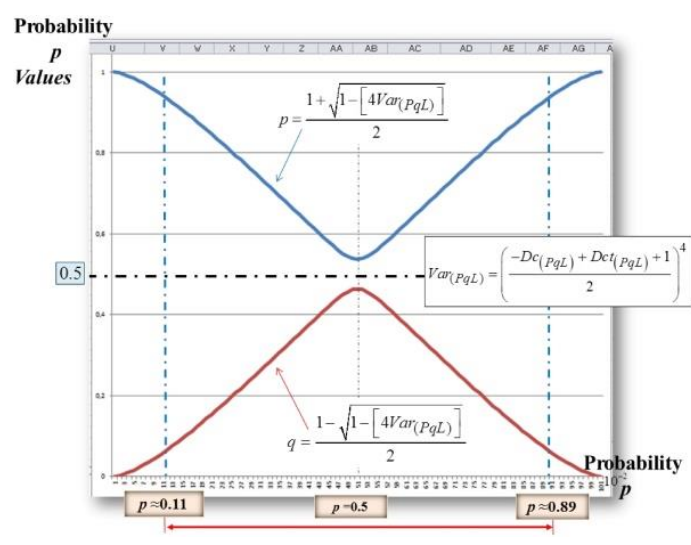

(b)

Fig. 12. (a) Results of the simulation of the PqL with the Heisenberg uncertainty principle using Eqs. (80) and (81). (b) Results of the simulation using Eqs. (84) and (85) to obtain probability values ranging from 0 to 1.0. 3.6 Results related to the Representation of the Division and Distance between Particles

Representations of the divisions and distances between particles in the paraconsistent model of the atom can be made by including factors in the probabilistic equations of the degree of certainty. Thus, a division and spacing between particles can be made by changing the equations of energies in Quadrant I of the interlaced bilattice of the PqL. These modifications in Quadrant I will be expanded to the three other quadrants through the negation, complement, and conflation operators. In this procedure, the equations of the degree of contradiction are left unchanged and a constant value is inserted into the equations of the degree of certainty, which we will call the distancing factor $\left(D_{F}\right)$. This causes a change of the values and locations of the probabilistic trajectories of the logical states. The new equations generated will have the same values for the degrees of evidence, which is represented by the Shannon entropy, and simulate other particles with different distances from each other, which depend on the value of $D_{F}$ inserted into the equation. The inclusion of the constant $D_{F}$ enables orbital paths or paths to be chosen in the model to simulate the bonding between atoms and other studies related to elementary particle set.

Fig. 13(a) shows the simulation of two particles in separation of $D_{F}=3$, and Fig. 13(b) shows the simulation of two particles in separation of $D_{F}=1$. 


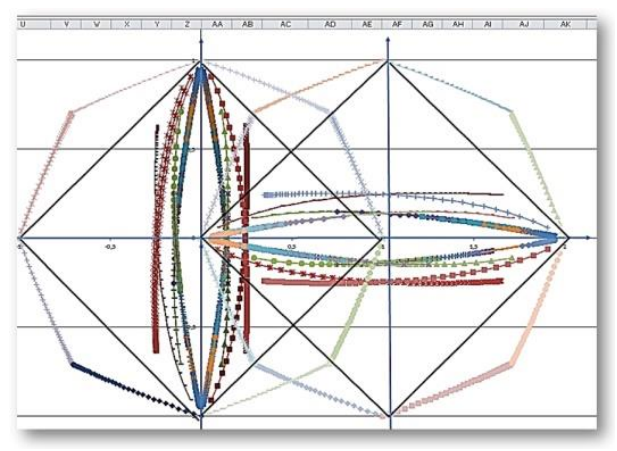

(a)

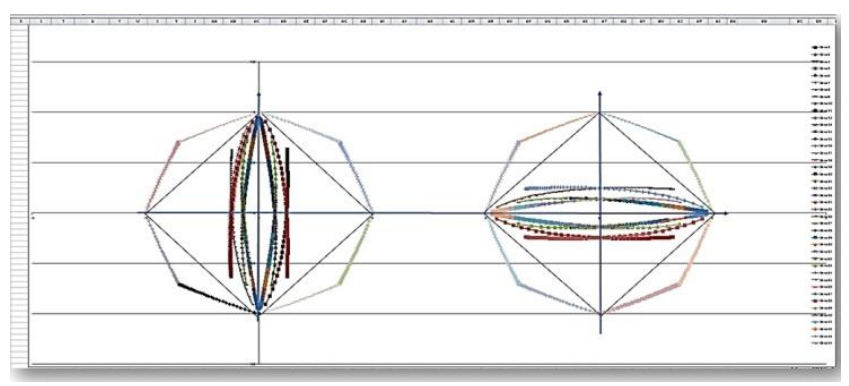

(b)

Fig. 13. (a) Simulation of two particles in separation of the $D_{F}=3$. (b) Simulation of two particles in separation of the $D_{F}=1$.

\subsection{Simulation Results with Energy Layer Values for the Hydrogen Atom}

As discussed previously, the degree of certainty $D c_{(p)}$ represents the differential energy in the representation of the paraquantum logical state. Thus, by multiplying each value of the degree of certainty of the energy obtained in each of the layers of the model by the value of the energy of the fundamental layer of the atom, the energies of each of the layers are determined. As expressed in Eq. (24), with the degree of favorable evidence $\mu_{(P q L) E 2}=H_{(s) P q L}$ and the degree of unfavorable evidence $\lambda_{(P q L) E 2}=H_{(s) P q L} \sqrt{\frac{H_{(s) P q L}}{2}}$, the degree of certainty for the energy level $E_{2}$ can reach its maximum value $D c_{(P q L) E 2}=\left(1-\frac{1}{\sqrt{2}}\right)$. To linearize the function of the degree of certainty and to regard its value as the differential energy between layers, we will use as the multiplying factor a value corresponding to the inverse of the normalized Planck constant raised to the cube, as follows: FactorDc $c_{(P q L) \text { Elinear }}=\frac{1}{\hbar^{3}} \simeq 0.85$. For the energy between layers 1 and 2 , the corresponding maximum multiplicative value will be FactorEnergy ${ }_{E n 1 E n 2}=\frac{1}{\hbar^{3}}\left(1-\frac{1}{\sqrt{2}}\right) \simeq 0.25$. This factor was adjusted to obtain the energies of the layers for the hydrogen atom, as will be discussed in the subsequent section.

\subsubsection{Linearization of the Energy Layer Values for the Hydrogen Atom}

Through the energy equations of the layers of the paraconsistent model of the atom, the simulations of the hydrogen atom were conducted. With fundamental energy of $13.6 \mathrm{eV}$, in the hydrogen atom, the differential energy between the fundamental layer and the other layers can be computed in linearized mode.

From Eq. (35), a linearizing factor of $\frac{1}{\hbar^{3}}$ can be applied to the degree of certainty to determine the differential energy of the second layer with constant value, as follows:

$$
\operatorname{Energy}_{(P q L) E 2}=\left[\frac{1}{\hbar^{3}}\left(H_{(s) P q L}-H_{(s) P q L} \sqrt{\frac{H_{(s) P q L}}{2}}\right)\right] \times 13.6 \mathrm{eV} .
$$

From Eq. (43), the differential energy between layers 2 and 3 can be computed using linearizing factor multiples of $\frac{1}{\hbar^{3}}$, as follows: 


$$
\operatorname{Energy~}_{(P q L) E 3}=\frac{1}{\hbar^{5}}\left[\frac{1}{\hbar^{6}} \sqrt{\frac{H_{(s) P q L}}{2}}-\left(\frac{1}{\hbar^{6}} \sqrt{\frac{H_{(s) P q L}}{2}} \times \sqrt{\frac{1}{\hbar^{6}} \sqrt{\frac{H_{(s) P q L}}{2}}}\right)\right] \times 13.6 \mathrm{eV}
$$

From Eq. (51), the differential energy between layers 3 and 4 can be computed using linearizing factor multiples of $\frac{1}{\hbar^{3}}$, as follows:

$$
\operatorname{Energy}_{(P q L) E 4}=\frac{1}{\hbar^{16}}\left[\frac{1}{\hbar^{6}} \sqrt{\frac{1}{\hbar^{6}} \sqrt{\frac{H_{(s) P q L}}{2}}}-\left(\frac{1}{\hbar^{6}} \sqrt{\frac{1}{\hbar^{6}} \sqrt{\frac{H_{(s) P q L}}{2}}} \times \sqrt{\frac{1}{\hbar^{6}} \sqrt{\frac{1}{\hbar^{6}} \sqrt{\frac{H_{(s) P q L}}{2}}}}\right]\right] \times 13.6 \mathrm{eV} .
$$

From Eq. (59), the differential energy between layers 4 and 5 can be computed using linearizing factor multiples of $\frac{1}{\hbar^{3}}$, as follows:

$$
D c_{n d(P q L) E 5}=\frac{1}{\hbar^{25}}\left[\frac{1}{\hbar^{6}} \sqrt{\frac{1}{\hbar^{6}} \sqrt{\frac{1}{\hbar^{6}} \sqrt{\frac{H_{(s) P q L}}{2}}}}-\left(\frac{1}{\hbar^{6}} \sqrt{\frac{1}{\hbar^{6}} \sqrt{\frac{1}{\hbar^{6}} \sqrt{\frac{H_{(s) P q L}}{2}}}} \times \sqrt{\frac{1}{\hbar^{6}} \sqrt{\frac{1}{\hbar^{6}} \sqrt{\frac{1}{\hbar^{6}} \sqrt{\frac{H_{(s) P q L}}{2}}}}} \times\right] \times 13.6 \mathrm{eV} .\right.
$$

From Eq. (68), the differential energy between layers 5 and 6 can be computed using linearizing factor multiples of $\frac{1}{\hbar^{3}}$, as follows:

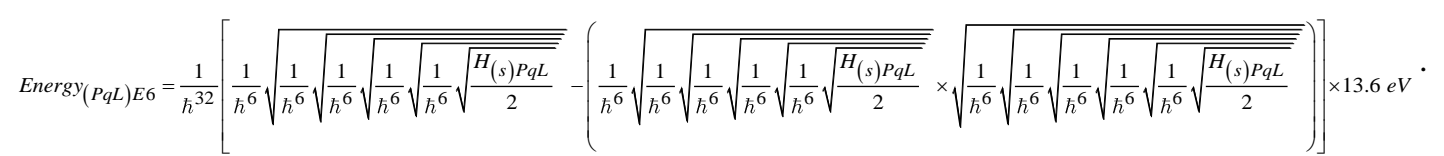

These procedures for PqL equations can be continued for $n$ layers of the paraconsistent model applied to the hydrogen atom.

Fig. 14(a) shows the simulation results for energy layers in the degenerate states of the hydrogen atom in the paraconsistent model of the atom. Fig. 14(b) shows the results of the paraconsistent functions of the energies obtained from the hydrogen atom using linearization.

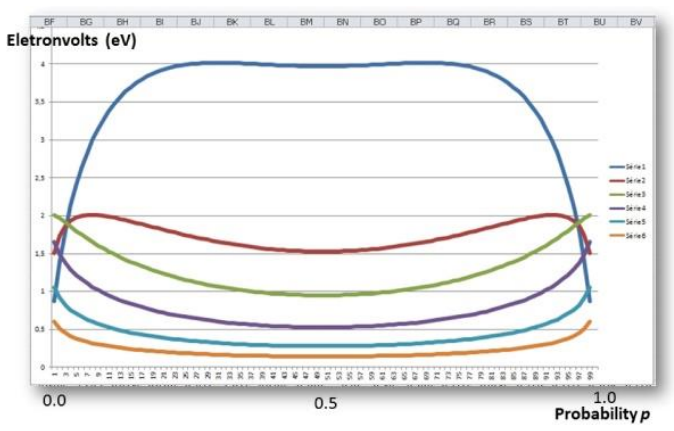

(a)

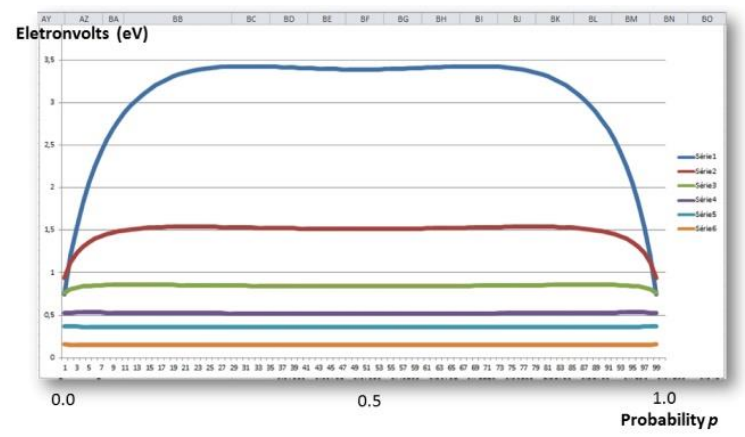

(b)

Fig. 14. (a) Energy of the layers in the paraconsistent model of the atom in the representation using Eqs. (33) to (70). (b) Energy of the layers in the degenerate state of the hydrogen atom in the paraconsistent model of the atom in the representation using linearization and Eqs. (86) to (90).

\subsection{Practice Example Results of the Paraconsistent Model of the Atom}

The paraconsistent model of the atom can be used in several knowledge domains. Moreover, paraconsistent logical algorithms have been successfully used to discriminate signals obtained by Raman spectroscopy (see references [26] and [28]). Data from Raman spectroscopy are obtained through vibrational processes of 
molecules involving laser application and capturing responses in the form of energy pulses. The obtained Raman information is related to the spectral lines that are provided as frequency-dominated Raman shifts or wavenumbers expressed in $\mathrm{cm}^{-1}$. Given the characteristics of the Raman spectroscopy signals, we can investigate experimentally how the paraconsistent model of the atom responds to variations in a particular wavelength range.

This experimental simulation consisted in applying the normalized values of Raman intensity in the equation of the degree of favorable evidence presented in Eq. (23). In this application, the values of probability $p$ are replaced in the Shannon entropy function by the complement of the normalized values of Raman intensity (1-I Raman $)$ throughout the spectrum of the sample.

$$
H_{(s) P q L}=-\hbar \pi[p \log p+(1-p) \log (1-p)] \rightarrow H_{(s) P q L}=-\hbar \pi\left[I_{\text {Raman }} \log I_{\text {Raman }}+\left(1-I_{\text {Raman }}\right) \log \left(1-I_{\text {Raman }}\right)\right] \text {. As }
$$

$\mu_{(P q L)}=H_{(s) P q L}$, the degree of evidence, which was probabilistic, was modified and became the degree of evidence of Raman intensity with energy characteristics due to the inclusion of the normalized Planck constant.

Fig. 15(a) shows two Raman spectroscopy signals in the 400 to $1,400 \mathrm{~cm}^{-1}$ range, which were recorded from the lubricating mineral oil sample. The first spectrum (Type 1) is related to the Raman data of normal lubricating mineral oil, and the second spectrum (Type 2) shows the Raman data of non-normal lubricating mineral oil. Type 2 (non-normal) lubricating mineral oil had its temperature controlled and maintained at approximately $127.5^{\circ} \mathrm{C}$ for $8 \mathrm{~h}$ and cooled to room temperature to obtain the Raman sample. The two Raman data spectra were applied to the paraconsistent model of the hydrogen atom and analyzed through the representation of the energy levels of the layers presented in this work (Eqs. (86) to (90)).

Fig. 15(b) shows the simulation results obtained the energy levels of the six layers in the paraconsistent hydrogen atom. In a superficial analysis, it is verified that the spectrum of normal lubricating mineral oil (Type 1) presents variations in the layers of the atom at several wavelengths. Some parts of the spectrum of non-normal lubricating mineral oil (Type 2) exhibits few energy variations in the layers of the atom considering the spectrum range investigated. 


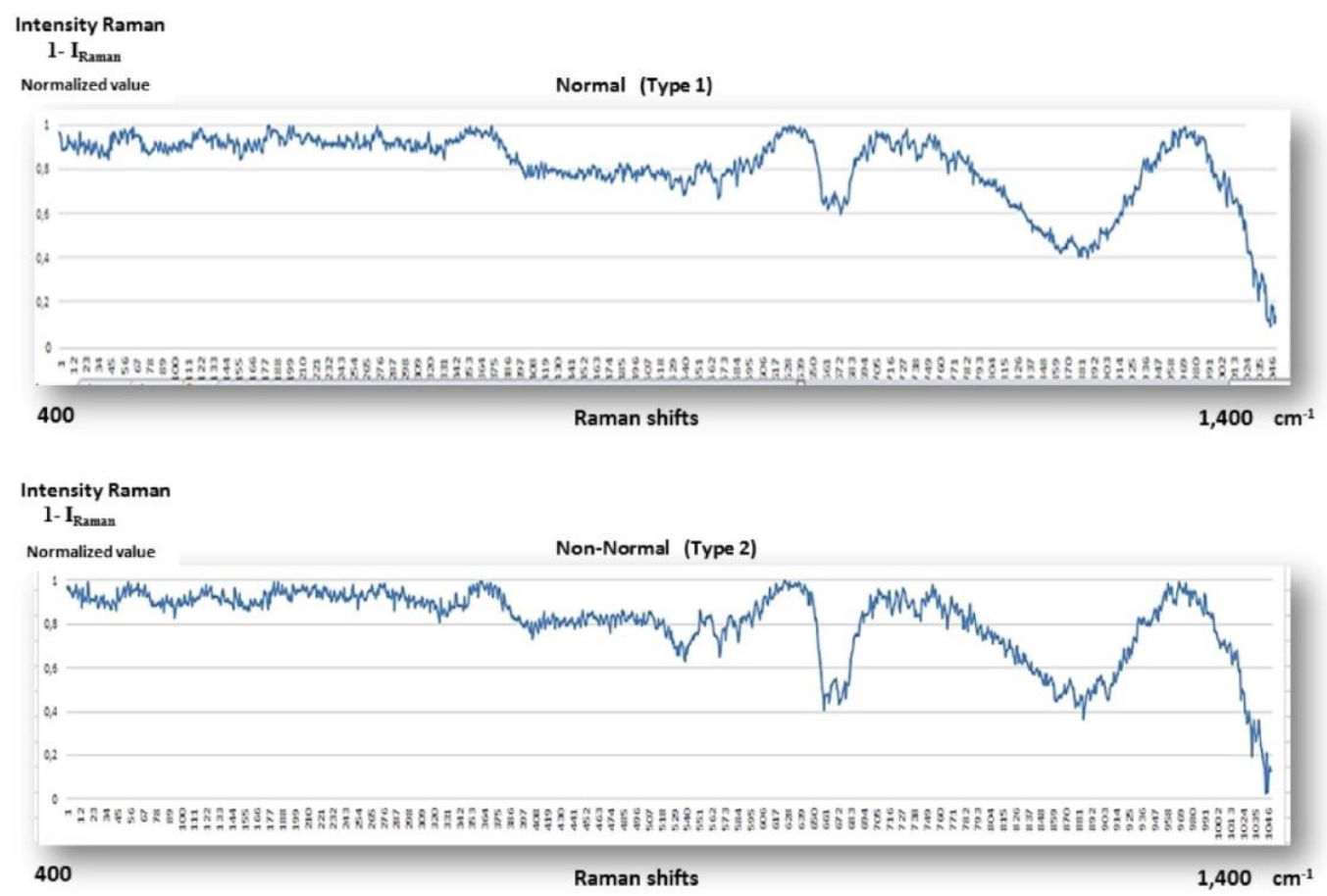

(a)

Energy levels in the layers of paraconsistent hydrogen atom
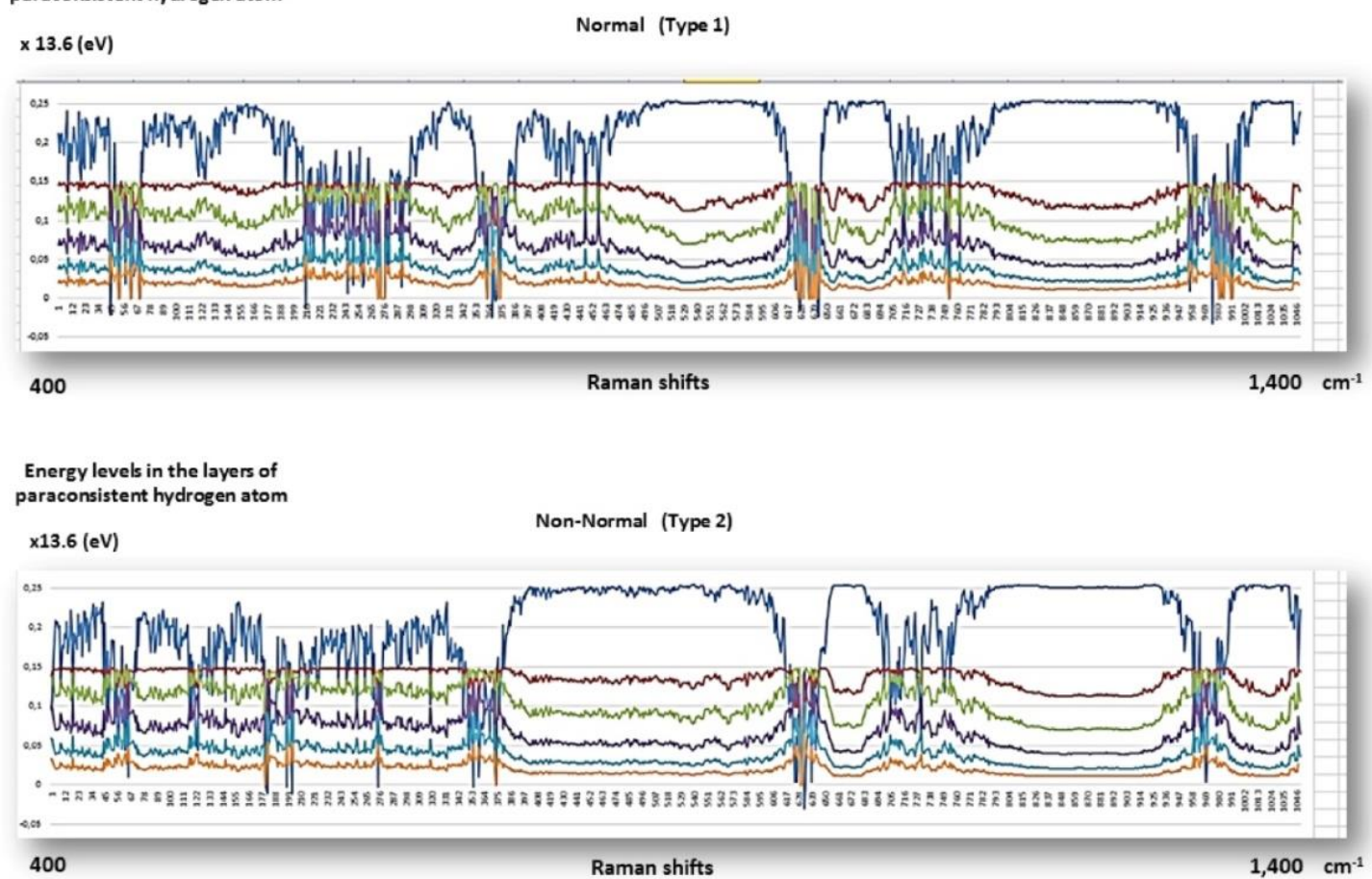

(b)

Fig. 15. (a) Raman spectroscopy signals from the lubricating mineral oil sample considered normal (Type 1) and non-normal (Type 2; heated for a preset time) (in 1- $\mathrm{I}_{\text {Raman }}$ normalized values). (b) Simulation results obtained for the energy levels of the six layers in the paraconsistent hydrogen atom.

\section{Discussion}


The equations presented in this work, as well as the method of obtaining them through interpretations of the interlaced bilattice of the PqL, followed the fundamentals of PAL2v, where the degree of favorable evidence $\mu$ must be accompanied by the degree of unfavorable evidence $\lambda$ to form the annotation. In the same manner, the degree of certainty $D c$ must be accompanied by the degree of contradiction $D c t$ to form the paraconsistent logical state. In Fig. 6(a), the graphs of the results obtained by simulations with Shannon entropy show how the variation of probability $p$ creates the path of logical states within the interlaced bilattice of the PqL. Notably, the correlation value of 0.5 between the curves of the degrees of evidence is the point that defines the boundaries ( $p$ $=0.11$ and $p=0.89$ ) between the evolution of the states in a balanced quantum system and the collapse of the wave function with the definition of false or true.

Fig. 6 (b) shows the unit value of the modulus of the internal vector $\mathrm{P} \psi_{\text {int }}$ that moves to create a geometric arc. The limits of its slope are defined by the equations of the Shannon entropy, and its movement with the two external vectors created by the complementarity of values indicates the uncertainty in the directions of the movement of these vectors.

The paraconsistent model of the atom presents the uncertainties that lead to incompleteness in the measurements, which is expected of a quantum system. This was demonstrated in the results of the simulations shown in Figs. 7(a) and 7(b), where the movements of the external vectors are antagonistic.

In Figs. 8 and 9, the results show the models separated by the reference of two observers. This condition is important for the quantum representations capable of being considered by the paraconsistent model of the atom. Fig.10 shows the simulation results for the complete paraconsistent model of the atom, as well as its projection of the state vectors in the imaginary $y$-axis of the contradiction and in the real $x$-axis in the case of an effected measurement. It was demonstrated how the actions of the negation, complementation and conflation operators applied to the logical states enabled the expansion of the probability values. From the analysis, the results obtained by the application of the operators to the logical states of Quadrant I showed that the trajectories of the particles in the layers appear simultaneously in two directions, that is, clockwise and counterclockwise.

Figs. 11(a) and 11(b) show the simulation results of the wave functions for the fundamental energy layer and the wave functions for the other energy layers. These results verified the limits that define the evolution of the states. Fig. 12(a) illustrates the validation of the results of the simulation with the Heisenberg uncertainty principle in the PqL, and Fig. 12(b) shows that is possible to obtain the value of the probability $p$ considering only the values of the degrees of certainty and contradiction. With these simulations, quantum concepts, such as probability density and wave function, were well founded by the equations and probabilistic functions in the PqL [40]. This representation, where the energy represented by the degrees of certainty and contradiction yields probability values, presents the paraconsistent model of the atom as in reality.

The results presented in Fig. 13 show the possibility of analyzing the paraconsistent model of the atom and other concepts of quantum mechanics, such as entanglement and teleportation.

In Fig. 14, the energies of the orbital layers represented by the pairs of values are well delineated in the representation of the hydrogen atom with values close to those obtained by the Bohr model.

On the basis of the results presented in Fig. 14, the experiments with Raman spectroscopy were elaborated, the results of which are presented in Fig. 15. A visual analysis of the results shown in Fig. 15(b) indicates that it is possible to verify the differences in energy variations between a normal lubricating mineral oil (Type 1) and a non-normal lubricating mineral oil (heated at $127.5^{\circ} \mathrm{C}$ for $8 \mathrm{~h}$; Type 2). Therefore, this technique using PqL can be useful for signal analysis and verification of material properties at atomic levels. 
In general, the simulation results define the probabilistic characteristics of the particle, where in quantum mechanics, before the measurement, any of the physical properties are always indefinite. In this manner, the configuration model exhibits the geometry of a sphere and can be described by means of equations that consider angular variables. A representation of the two planes can be made according to Fig. 12, where the orthogonality of the two planes forms an octahedron in which an analysis of the external and internal variables in the equations validates the variation of probability $[41,42]$. With the probability representation obtained through the Shannon entropy equation in which the normalized Planck constant $\hbar$ was introduced, we can derive the equations of the degrees of certainty and contradiction by applying the conditions used for a paraconsistent Bloch sphere as an example.

\section{Conclusions}

The paraconsistent model of the atom proposed in this work uses the foundations of the PAL and combines the concepts of the entropy of information theory with quantum mechanics. The proposed paraconsistent model of the atom, which applied the interlaced bilattice of the $\mathrm{PqL}$, showed a convincing geometric aspect for the atomic particle. Thus, it was possible to demonstrate well-adjusted fundamentals for the phenomena of quantum physics. In spite of the impossibility of covering all quantum phenomena in this work, the equations obtained by the analyses provide the characteristics of symmetry, recurrence, and superposition of states. The entanglement concept can be expressed in the form of the division obtained through the distance factor $D_{F}$ used in the equations of the degrees of certainty. The quantum phenomenon of the superposition of states is explicitly expressed through the equations and the representation in which two observers are active. This procedure, which was conducted through the simultaneous use of equations and analysis of an observer in the vector of the base $X$ and an observer in the vector of the base $Y$, is similar to the quantum theory. The model presented in this work is innovative and opens a field of in-depth investigations of different conditions and the effects originating from interpretations of the model under diverse conditions and dimensions. All of the equations exhibit good computability and ensure that all of the procedures can be presented in matrix and algorithmic forms. Notably, in the paraconsistent model of the atom presented in this work, no equation results in a defined value. All of the equations used in the simulations were probabilistic functions that resulted in indefinite trajectories of the paraquantum logical states. Even the direction and orientation of the orbital trajectories shown in the result graphics are indefinite because they are always antagonistic to one another. In its application to Raman spectroscopy data, the paraconsistent model of the atom showed versatility and a good representation of the energy variations at the atomic level of hydrogen. These results enable us to make new explorations of the use of the proposed model with the presented concepts, such as the linearization of the energy values in the lower layers of the hydrogen atom and for other atomic models. This work demonstrated that the use of the Shannon entropy with the inclusion of the normalized Planck constant $\hbar$ in a paraconsistent model is an excellent method of modeling quantum systems. In the future, new simulation procedures will expand the application possibilities of the paraconsistent model of the atom in the areas of computation, quantum logic gates, quantum systems for signal recognition, and quantum cryptography, among others.

Supplementary Materials: The data that support the plots within this paper and other findings of this study are available in additional material and from the corresponding author.

Author Contributions: J.I. carried out the experiments, did the analysis of the results, wrote the paper and prepared the illustrations. 


\section{Acknowledgments:}

The author acknowledges ISESC - Instituto Superior de Educação Santa Cecilia for the financial support for this research.

The author would like to acknowledge L. Silveira Jr. for Raman spectroscopy data obtained by FAPESP (São Paulo Research Foundation) process no. 2009/01788-5.

Conflicts of Interest: The author declares no competing interests.

\section{References}

[1] Kragh, H.; Rigden, J. S. Niels Bohr and the Quantum Atom: The Bohr Model of Atomic Structure 1913-1925. American Journal of Physics 81(3):237-238, 2013. DOI: 10.1119/1.4771884

[2] Heilbron, J. L.; Kuhn, T. S. The genesis of the Bohr atom. Historical Studies in the Physical Sciences 1, 211-290, 1969. DOI: $10.2307 / 27757291$

[3] Auletta, G. Foundations and Interpretation of Quantum Mechanics, World Scientific, 2001.

[4] Bohm, D. A. Suggested Interpretation of the Quantum Theory in Terms of "Hidden" Variables, Phys. Rev. 85, 166-193, 1952.

[5] Feynmann, R. P. The Feynman Lectures on Physics, Volume 3, Addison Wesley, 1963.

[6] Feynmann, R. P. Simulation physics with Computers, Journ. Th. Phys. 21, 467, 1982.

[7] Dirac, P. A. M. The Principles of Quantum Mechanics, 16 Oxford University Press, 1958.

[8] Born, M. The statistical interpretation of quantum mechanics - Nobel Lecture, December 11, 1954.

[9] Nielsen,M. A.; Chuang, I. L. Quantum Computation and Quantum Information. Cambridge University Press, 2000.

[10] Da Costa, N. C.; de Ronde, A. C. Found Phys 43: 845, 2013, DOI: 10.1007/s10701-013-9721-9.

[11] Dalla Chiara M. L.; Giuntini, R. Unsharp quantum logics, Foundations of Physics 24, 1161-1177, 1994.

[12] Dalla Chiara, M. L.; Giuntini, R.; Greechie, R. Reasoning in Quantum Theory, Kluwer Academic Publishers, Dordrecht, 2004.

[13] Engesser, K.; Gabbay, D. M.; Lehmann, D. A. New Approach to Quantum Logic (Studies in Logic book 8). 200p. ISBN-13: 978-1904987536. College Publications, 2007.

[14] Aspray, W. The mathematical reception of the modern computer: John von Neumann and the institute for advanced study computer. In Studies in the History of Mathematics, E. R. Phillips, Ed., v. 26. MAA, Washington DC, pp. 166-194, 1987.

[15] von Neumann, J. Applications of the ergodic hypothesis. Proceedings of the National Academy of Sciences 18, 263-266. Reprinted in (Taub 1961a) 13. 1932.

[16] Bruckmann, G. Weber, W. Eds. Contributions to the von Neumann Growth Model (New York), Springer Verlag, 1971.

[17] Vedral, V. The role of relative entropy in quantum information theory. Rev. Mod. Phys. 74, 197, 2002

[18] Nielsen, M. A. , Chuang, I. L. Quantum Computation and Quantum Information. Massachusetts Institute of Technology, 702 pages - 2010, ISBN: 9781107002173

[19] Abe, J. M.; Nakamatsu, K.; Akama, S.; Da Silva Filho, J. I. The Importance of Paraconsistency and Paracompleteness in Intelligent Systems. In: Czarnowski I., Howlett R., Jain L. (eds) Intelligent Decision Technologies 2017. IDT 2017. Smart Innovation, Systems and Technologies, 73. Springer, Cham, (2018).

[20] Da Costa, N. C. A. On the theory of inconsistent formal systems. NotreDame Journal Form Log, 15(4), 497-510. 1974. DOI: http://dx.doi.org/10.1305/sdjfl/1093891487 
[21] Da Costa, N. C. A.; Marconi, D. An overview of Paraconsistent logic in the 80's., The Journal of Non-Classical Logic, 6, 5-31, 1989.

[22] Subrahmanian, V. S. On the semantics of quantitative logic programs. Proc. 4th. IEEE Symposium on Logic Programming, Computer Society Press, Washington D.C, 1987.

[23] Abe, J. M.; Akama, S.; Nakamatsu, K. Introduction to Annotated Logics. Springer, Heidelberg, 2016

[24] Blair, H. A.; Subrahmanian, V. S. Paraconsistent Logic Programming, Theoretical Computer Science 68, 135-154, 1989.

[25] Belnap, N. A. Useful four-valued logic. In J. M. Dunn \& G. Epstein (orgs.), Modern uses of multiple-valued logic (p. 8-37). Dordrecht: Reidel. 1977b.

[26] Garcia, D. V.; Da Silva Filho, J. I.; Silveira Jr, L. et al., Analysis of Raman spectroscopy data with algorithms based on paraconsistent logic for characterization of skin cancer lesions. Vibrational Spectroscopy, 103, 2019. DOI: 10.1016/j.vibspec.2019.102929

[27] Da Silva Filho, J. I. Métodos de interpretação da lógica Paraconsistente Anotada com anotação de dois valores - LPA2v com construção de algoritmo e implementação de Circuitos Eletrônicos. Doctoral Thesis (in portuguese), Polytechnic School of the University of São Paulo, POLI / USP São Paulo, Brazil, p. 115, 1999.

[28] Da Silva Filho, J. I.; Nunes, V. C.; Garcia, D. V.; Mario, M.C.; Giordano, F.; Abe, J. M.; Pacheco, M. T. T.; Silveira Jr., L. Paraconsistent analysis network applied in the treatment of Raman spectroscopy data to support medical diagnosis of skin cancer. Med. Biol. Eng. Comput. 54, 1-15,_2016). DOI: $10.1007 / \mathrm{s} 11517-016-1471-3$

[29] Coelho, M. S.; Da Silva Filho, J. I.; Côrtes, H. M. et al. Hybrid PI controller constructed with paraconsistent annotated logic. Control Engineering Practice, 84, 112-124, 2019. DOI: 10.1016/j.conengprac.2018.11.007

[30] Da Silva Filho J. I.; Lambert-Torres, G.; Abe J. M. Uncertainty Treatment Using Paraconsistent Logic Introducing Paraconsistent Artificial Neural Networks. IOS Press, 328 Volume 211- Frontiers in Artificial Intelligence and Applications, Amsterdam, Netherlands, (2010).

[31] Da Silva Filho, J. I. A Probabilistic Paraconsistent Logical Model for Non-Relativistic Quantum Mechanics Using Interlaced Bilattices with Conflation and Bernoulli Distribution. Journal of Quantum Information Science, 7, 89-124. 2017. DOI: 10.4236/jqis.2017.73009

[32] Da Silva Filho, J.I. Undulatory Theory with Paraconsistent Logic (Part I): Quantum Logical Model with Two Wave Functions. Journal of Quantum Information Science, 6, 143-180, 2016. DOI: 10.4236/jqis.2016.63012

[33] Da Silva Filho, J.I. Undulatory Theory with Paraconsistent Logic (Part II): Schrödinger Equation and Probability Representation. Journal of Quantum Information Science, 6, 181-213, 2016. DOI: 10.4236/jqis.2016.63013

[34] Belnap, N. How a computer should think. In G. Ryle (ed.), Contemporary Aspects of Philosophy (pp. 30-55). Stocks_eld: Oriel Press. 1977a.

[35] Ginsberg, M. L. Multivalued logics: A uniform approach to inference in artificial intelligence, Computational Intelligence 4, 265-316, 1988.

[36] Shannon, C. E. Bell Syst. Tech. J. $27 \quad \mathbf{1 9 4 8} 379\{423$ and 623\{656, DOI:10.1002/j.1538-7305.1948.tb01338.x.

[37] Shannon, C. E.; Weaver W. The Mathematical Theory of Communication, Illini Books, Illinois, 1949. 
[38] Tempesta, P. Beyond the Shannon-Khinchin formulation: The composability axiom and the universal-group entropy. Annals of Physics, 365, 180-197, 2016. DOI: 10.1016/j.aop.2015.08.013

[39] Marcos D., Widmer P., Rico E., Hafezi M., Rabl P., Wiese U.-J., Zoller P., Two-dimensional lattice gauge theories with superconducting quantum circuits. Annals of Physics, 351, 634-654, 2014. DOI: 10.1016/j.aop.2014.09.011

[40] Grinstead C. M., Snell J. L. Introduction to probability. Providence, RI, American Mathematical Society, 1997.

[41] Rastegin A. E. On entropic uncertainty relations in the presence of a minimal length. Annals of Physics, 382, 170-180, 2017. DOI: 10.1016/j.aop.2017.04.014

[42] Ord G. N. Quantum mechanics in a two-dimensional spacetime: What is a wavefunction?. Annals of Physics, 324(6), 1211-1218, 2009. DOI: 10.1016/j.aop.2009.03.007 